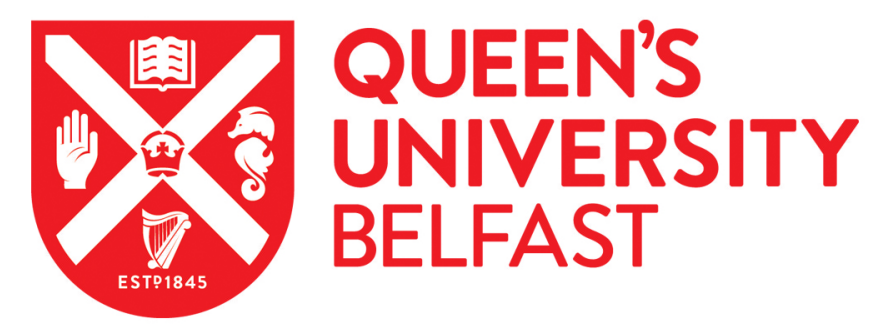

\title{
Modeling Circuits with Arbitrary Topologies and Active Linear Multiports using Wave Digital Filters
}

Werner, K. J., Bernardini, A., Smith, J., \& Sarti, A. (2018). Modeling Circuits with Arbitrary Topologies and Active Linear Multiports using Wave Digital Filters. IEEE Transactions on Circuits and Systems I: Regular Papers, 65(12), 4233-4246. https://doi.org/10.1109/TCSI.2018.2837912

\section{Published in:}

IEEE Transactions on Circuits and Systems I: Regular Papers

\section{Document Version:}

Peer reviewed version

Queen's University Belfast - Research Portal:

Link to publication record in Queen's University Belfast Research Portal

\section{Publisher rights}

(c) 2018 IEEE.

This work is made available online in accordance with the publisher's policies. Please refer to any applicable terms of use of the publisher.

\section{General rights}

Copyright for the publications made accessible via the Queen's University Belfast Research Portal is retained by the author(s) and / or other copyright owners and it is a condition of accessing these publications that users recognise and abide by the legal requirements associated with these rights.

Take down policy

The Research Portal is Queen's institutional repository that provides access to Queen's research output. Every effort has been made to ensure that content in the Research Portal does not infringe any person's rights, or applicable UK laws. If you discover content in the Research Portal that you believe breaches copyright or violates any law, please contact openaccess@qub.ac.uk. 


\title{
Modeling Circuits with Arbitrary Topologies and Active Linear Multiports using Wave Digital Filters
}

\author{
Kurt James Werner, Member, IEEE, Alberto Bernardini, Student Member, IEEE, \\ Julius O. Smith III, Member, IEEE, and Augusto Sarti, Senior Member, IEEE
}

\begin{abstract}
The Wave Digital Filter (WDF) technique derives digital filters from analog prototypes which classically have been restricted to passive circuits with series/parallel topologies. Since most audio circuits contain active elements (e.g., opamps) and complex topologies, WDFs have only had limited use in Virtual Analog modeling. In this article we extend the WDF approach to accommodate the unbounded class of nonseries/parallel junctions which may absorb linear multiports. We present four Modified-Nodal-Analysis-based scattering matrix derivations for these junctions, using parametric waves with voltage, power, and current waves as particular cases. Three derivations afford implementations whose cost in multiplies are lower than multiplying by the scattering matrix. Negative port resistances may be needed in WDF modeling of active circuits, restricting the WDF to voltage or current waves. We propose two techniques for localizing this restriction. Case studies on the Baxandall tone circuit and a "Frequency Booster" guitar pedal demonstrate the proposed techniques in action.
\end{abstract}

Index Terms-Wave Digital Filters, Virtual Analog, Physical Modeling, Circuit Simulation, Active Circuits, Scattering.

\section{INTRODUCTION}

$\mathbf{W}$ AVE Digital Filters (WDFs) [1] are digital filters with structures based on analog reference circuits or other lumped systems (e.g. mechanical or acoustical [2], [3]) whose simulation parameters are based directly on the physical reference system quantities. Alfred Fettweis invented WDFs in the early 1970s [4], [5]; he and others brought the field to a high level of maturity by the mid 1980s [1]. The key WDF concepts are computability and passivity. As their signal variables, WDFs use wave quantities that are linear combinations of port voltages and currents from classical network theory [6], parametrized by port resistances. By choosing a proper discretization (classically, the bilinear transform) for reactances (capacitors, inductors), the reference circuit's passivity is preserved in the WDF. For fixed-point implementation, the scattering behavior of 3-port "adaptors" [7] can be implemented with incremental passivity, i.e., where any numerical error corresponds to a small loss of energy [8]. Early WDF theory covered ladder and lattice filters [9], [10] with topologies composed entirely of 3-port series/parallel

K. J. Werner is with the Sonic Arts Research Centre (SARC), School of Arts, English and Languages, Queen's University Belfast; 4 Cloreen Park, Belfast BT9 5HN, U.K. (e-mail: k.werner@qub.ac.uk).

A. Bernardini and A. Sarti are with the Dipartimento di Elettronica, Informazione e Bioingegneria (DEIB), Politecnico di Milano; Piazza L. Da Vinci 32, 20133 Milano, Italy (e-mail: [alberto.bernardini, augusto.sarti]@ polimi.it)

J. O. Smith III is with the Center for Computer Research in Music and Acoustics (CCRMA), Stanford University; 660 Lomita Drive, Stanford, California 94305, USA (e-mail: jos@ccrma.stanford.edu)

Manuscript received...; revised.... connections (Fig. 1a-1b), whose good sensitivities translated to good numerical properties (accuracy and dynamic range) in the WDF [1], [8]. For comprehensive reviews of WDF principles, the reader is directed to, e.g., [1], [2].

The music technology community began to engage with WDFs in 1987, when Smith noted the connection to his Digital Waveguide Filters, which use wave variables, scattering junctions, and delay lines to model acoustic propagation [11]. Starting in the 1990s, Sarti et al. advanced WDF theory, applying it in particular to modeling acoustic systems [12]-[14]. Building on work by Fettweis and Nitsche [15], Bilbao used WDFs to model acoustical distributed systems [16]. Later, WDFs were adopted in Virtual Analog (VA) research. VA modeling recreates analog musical effects (amplifiers, effect pedals, etc.) and instruments (synthesizers, drum machines, etc.) in the digital domain. Early VA WDF models include tube amps [17], simple guitar circuits [18], and ad hoc models of envelope followers and oscillators [2].

The primary goal of VA is real-time transient simulation of audio circuits with any possible control setting (potentiometers, etc.). WDFs are well-aligned with this goal, since they are simulations parametrized by component values, whose explicit computation has an efficient, fixed cost. Furthermore, WDFs typically have a fixed time step (although variable-step extensions exist [19], [20]), matching the convention of audio signal processing. Mainstream circuit simulation packages like SPICE [21] inherently perform variable time step transient simulations out of real time using global iterative solvers, making them a poor match to the needs of VA.

Unfortunately WDF modeling has had limitations in VA. Most audio circuits contain components (e.g. op-amps, controlled sources, nullors [22], [23], multiple nonlinearities) and topologies (e.g. feedback, bridges [24]) that have not been systematized in WDF modeling. Here we review and point out limitations of traditional WDF structures. An overview of this article and how it helps to address these limitations follows.

\section{A. WDF Global Structure}

The global structure of a standard WDF is a rooted tree with adaptors and one-ports as nodes and port connections as edges. Classical series/parallel structures can be described by a Binary Connection Tree (BCT) [2], [14]. More generally we consider Connection Trees (CT) [25]. Every WDF element in the CT must be adapted, i.e., its upwards-facing (towards the root of the CT) port resistance must be chosen so that the reflected wave at that port does not depend instantaneously on 


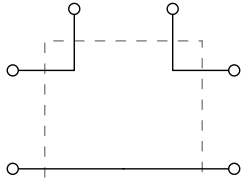

(a) Series

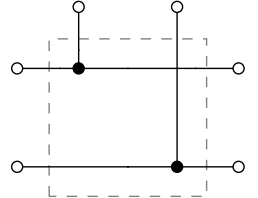

(b) Parallel.

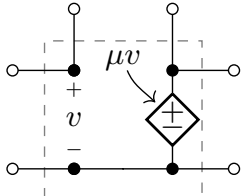

(c) Absorbed VCVS.
Fig. 1. Three examples of 3-port junctions.

the incident wave at that port. The only exception is the root element, which by definition has no upwards-facing port.

CTs for simple circuits can be found by inspection, but in general an automatable approach is better. For passive and reciprocal circuits, Fränken et al. proposed systematic generation of WDF CTs based on the SPQR tree from graph theory [26], representing classical 3-port series $(\mathcal{S})$ and parallel $(\mathcal{P})$ connections, $\mathcal{R}$-type rigid connections, and oneport circuit elements $(\mathcal{Q})$. There are an unbounded number of $\mathcal{R}$-type connections, some that are "pure" junctions (wire interconnections between port terminals) and some that involve absorbed nonenergic (passive and reciprocal) elements. For examples of this procedure, see the case studies $(\S \mathrm{IV})$.

\section{B. Scattering of Reciprocal Junctions}

To model $\mathcal{R}$-type connections as adaptors in a WDF CT, a procedure is needed for implementing their scattering behavior. For pure junctions (e.g. Figs. 1a-1b) or junctions with absorbed reciprocal elements (e.g. transformers), the method of Martens and Meerkötter [27] can be used. This method represents a reciprocal multiport on the graph-theoretic links and twigs basis and uses the orthogonality of the circuit and cutset matrices to derive voltage wave scattering behavior. This has been used for particular network synthesis reciprocal multiports sections including bridged-T [27], twin-T [28], Brune / Darlington C, and Darlington D [29]. A technique for efficient implementation is proposed in [30].

This approach has two major restrictions. First, it is only valid for reciprocal junctions. Unfortunately non-reciprocal and active circuits are ubiquitous in audio, where circuits are often designed around active elements such as controlled sources, op-amps, operational transconductance amplifiers, etc. Additionally, the method is restricted to voltage waves.

\section{Scattering of Non-Reciprocal Junctions}

For circuits with non-reciprocal junctions, we apply the CT generation procedure of [26], treating these junctions identically to reciprocal junctions. This yields $\mathcal{R}$-type junctions with absorbed active and non-reciprocal two-ports. An example is shown in Fig. 1c. To create a WDF model, the scattering behavior of these junctions must be derived. However, since non-reciprocal junctions do not have orthogonal circuit and cutset matrices, the method of [27] cannot be used.

A very general approach to finding a scattering matrix of junctions with complicated topologies and absorbed active elements was first proposed by Werner et al. in [31] and detailed further in [25]. This solution represents the incident wave at each port of a junction by a Thévenin equivalent, solves port currents using Modified Nodal Analysis [32], and forms reflected waves from the incident waves and port currents. This greatly expanded the class of circuits that could be modeled with WDFs. Audio circuits that were previously intractable using WDFs, including the op-amp-based tone stage from the Ibanez Tube Screamer [25], simplified analog drum machine voices [33], the Hammond Organ vibrato/chorus [34], [35], op-amp-based Sallen-Key filters [36], [37], operationaltransconductance-amplifier-based state variable filters [38], and filters based around linearized emitter followers [39] have been modeled using this approach.

This approach has two restrictions. First, the method is again only formulated in terms of voltage waves. Second, the method is expensive to compute-for an $N$-port $\mathcal{R}$-type adaptor, the scattering costs $N^{2}$ multiplies per sample. Since $\mathcal{R}$-type multiports have no upper bound on size, the cost can become prohibitive, especially for VA where real-time implementation is important. Classical series and parallel adaptors have realizations with far fewer than $N^{2}$ multiplies [1], suggesting that something similar should be possible for $\mathcal{R}$-type adaptors.

\section{Overview}

In this article, we expand the class of circuits that may be modeled using Wave Digital Filter principles to include active circuits with any topology, involving any number of linear multiports that may be active and non-reciprocal. This is accomplished by first pointing out that the SPQR decomposition of Fränken et al. (claimed to exclude active elements [26]) may indeed be applied to circuits with active elements.

Second, we develop a family of scattering matrix equations for $\mathcal{R}$-type multiports, accommodating a generalized class of waves which includes voltage, current, and power waves as particular cases. Four forms arise from combining two ways of representing incident waves and two approaches to solving for reflected waves and yield identical scattering matrices.

Third, by exploiting the form of these equations, the scattering relationship can be implemented without calculating the scattering matrix. Three forms usually have a reduced persample implementation cost in multiplies compared to matrix multiplication. This extends and generalizes the approach of [25] which was restricted to voltage waves and had no provisions for reducing the implementation cost of an $N$-port scattering operation below the nominal $N^{2}$ multiplies.

Finally, we address negative port resistances which may arise while adapting active multiports. Negative port resistances cause complex signal values that are not physically meaningful in a transient simulation, meaning that they portions of a WDF structure with negative port resistances are restricted to voltage or current waves. We propose two techniques for localizing this restriction: A two-port that allows WDF structures with different wave definitions to be interfaced, and an extension to the $\mathcal{R}$-type adaptor derivation that allows each port to have its own wave definition.

Baxandall tone circuit and a Frequency Booster guitar pedal case studies demonstrate the proposed method in action.

The rest of the article is organized as follows. In $\S I I$ the proposed method is developed. Two approaches to WDF 


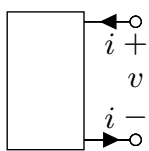

(a) One port

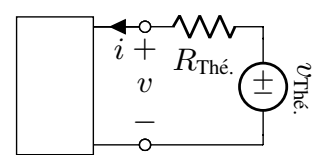

(b) Thévenin equivalent.

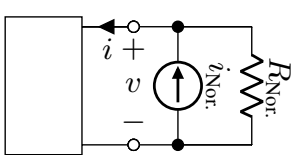

(c) Norton equivalent.
Fig. 2. One port and Thé./Nor. equivalents representing attached circuits.

structures with multiple wave types are presented in $\S I I I$. The two case studies are detailed in $\S \mathrm{IV}$. $\S \mathrm{V}$ concludes.

\section{SCATtERING OF ACtive Junctions}

Here, we propose methods for deriving the scattering behavior of junctions with any number of absorbed multiports, which may be active and non-reciprocal. We first define the types of junctions and waves that are considered. The incident waves (inputs) to the junction are represented by Thévenin/Norton equivalents, the junction's behavior is solved using Modified Nodal Analysis, and the reflected waves (outputs) are formed, yielding a family of four scattering matrix equations. Alternative scattering implementations are proposed, which are often less expensive than a simple multiply by the scattering matrix.

\section{A. Junction Definition}

Each junction has a number of ports, which are two-terminal connections to the junction with a positive and a negative terminal. Each port (Fig. 2a) is characterized by a voltage $v$ across it and a current $i$ through it. By convention, $i$ is defined as the current flowing into the positive terminal and is equal to the current flowing out of the negative terminal.

"Pure" (e.g. series or parallel) junctions are composed entirely of wire interconnections between their port terminals. A multiport junction may also involve internal two-ports, e.g., transformers, controlled sources, and nullors. This occurs when there is an external connection between the two ports of a multiport. Since many audio circuits are designed around feedback principles, this situation is extremely common.

In this article, we consider the wide class of junctions with $N>0$ ports and any number of absorbed linear two-port electrical elements, which may be active and non-reciprocal.

\section{B. Wave Definitions}

The standard way to represent the behavior of multiport junctions is through a matrix relationship between their port currents and voltages. However, attempting to build a discretetime simulation of a circuit by connecting many of these representations together will invariably cause instantaneous dependencies called "delay-free loops," making explicit computation impossible. For this reason, the WDF approach to circuit modeling adopts the use of wave variables.

In WDFs, voltage, power, and current waves are the most common. Voltage waves are usually used [1] and power waves have useful properties [16], [40]. The definitions are

$$
\begin{array}{lll}
a^{(\mathrm{v})}=v+R i & a^{(\mathrm{p})}=v / \sqrt{R}+\sqrt{R} i & a^{(\mathrm{i})}=v / R+i \\
b^{(\mathrm{v})}=v-R i & b^{(\mathrm{p})}=v / \sqrt{R}-\sqrt{R} i & b^{(\mathrm{i})}=v / R-i
\end{array}
$$

where $v, i \in \mathbb{R}$ are the port voltage and current, $a, b \in \mathbb{R}$ are the incident and reflected waves, and $R \in \mathbb{R} \backslash\{0\}$ is the port resistance. The superscripts (v), (p), (i) denote wave type (voltage, power, current) and are usually dropped for compactness. Although $R \in \mathbb{R}_{>0}$ is usually assumed due to the important role of passivity in traditional WDFs, negative port resistances may arise in adapting active junctions or negative impedances Energetic considerations for negative port resistances are given in [41].

To unify the traditional wave definitions, we introduce a new parametric wave definition and its inverse

$$
\begin{aligned}
& a^{(\rho)}=R^{\rho-1} v+R^{\rho} i \\
& b^{(\rho)}=R^{\rho-1} v-R^{\rho} i
\end{aligned} \longleftrightarrow \begin{aligned}
& v=R^{1-\rho}\left(a^{(\rho)}+b^{(\rho)}\right) / 2 \\
& i=R^{-\rho}\left(a^{(\rho)}-b^{(\rho)}\right) / 2
\end{aligned}
$$

where the parameter $\rho \in \mathbb{R}$ defines the wave type. In the case of a vector of $N$ ports, this relationship is given by

$$
\begin{aligned}
& \mathbf{a}^{(\rho)}=\mathbf{R}^{\rho-1} \mathbf{v}+\mathbf{R}^{\rho} \mathbf{i} \\
& \mathbf{b}^{(\rho)}=\mathbf{R}^{\rho-1} \mathbf{v}-\mathbf{R}^{\rho} \mathbf{i}
\end{aligned} \longleftrightarrow \begin{array}{r}
\mathbf{v}=\mathbf{R}^{1-\rho}\left(\mathbf{a}^{(\rho)}+\mathbf{b}^{(\rho)}\right) / 2 \\
\mathbf{i}=\mathbf{R}^{-\rho}\left(\mathbf{a}^{(\rho)}-\mathbf{b}^{(\rho)}\right) / 2
\end{array}
$$

with vector port quantities $\mathbf{v}, \mathbf{i}, \mathbf{a}, \mathbf{b} \in \mathbb{R}^{N \times 1}$ and a diagonal matrix of port resistances $\mathbf{R}=\operatorname{diag}\left(\left[R_{1}, R_{2}, \ldots, R_{N}\right]\right)$. In this article we will deal with the particular values of $\rho$ corresponding to the traditional wave definitions

$$
\rho=\left\{\begin{array}{ll}
1 & \text { voltage waves } \\
1 / 2 & \text { power waves } \\
0 & \text { current waves }
\end{array} .\right.
$$

New versions of traditional WDF elements can be derived by plugging (1) or (2) into the constitutive equations for an electrical element and solving for the reflected wave(s). A catalog is given in [39]; here we only point out a few properties. First, $\rho$ never affects port adaptation criteria, which depend on matching the incremental resistance of an electrical element, discretized companion model of a reactance, or adaptor. For linear immittances, $v$ and $i$ in (1) are proportional to $R^{\rho}$ and cancel out so that $\rho$ does not affect the signal flow. For current or voltage sources, $\rho$ acts as an input scaling. For multiports, $\rho$ changes the scattering matrix dramatically because the $R^{\rho}$ terms for different ports will not cancel out.

Traditionally, all ports in each junction and even the entire WDF have used the same wave definition. In $\S I I I$, we introduce techniques for accommodating multiple wave types within one WDF, allowing a greater degree of simulation flexibility.

\section{Representing Inputs}

To derive the scattering behavior of a general $\mathcal{R}$-type adaptor, we represent the incident wave at each port as a Thévenin (Thé.) or Norton (Nor.) equivalent circuit (Figs. 2b2c) which comprises an ideal source and a resistor.

The component values of these "instantaneous port equivalents" can be found by rewriting (1) to solve for $v$ or $i$

$$
v=R^{1-\rho} a-R i \quad \text { (3) } \quad i=R^{-\rho} a-R^{-1} v
$$

and recognizing that these describe Kirchhoff's Voltage Law (KVL) around the open port of a Thé. equivalent with $R_{\text {Thé. }}=$ $R$ and $v_{\text {Thé }}=R^{1-\rho} a$ or Kirchhoff's Current Law (KCL) at one terminal of a Nor. equivalent with $R_{\text {Nor. }}=R$ and $i_{\text {Nor. }}=$ $R^{-\rho} a$. The incident wave only affects the source; the wave definition is also embedded in the resistance's value. 
TABLE I

MODIFIED NODAL ANALYSIS STAMPS FOR ONE-PORT AND TWO-PORT ELEMENTS RELEVANT TO THE PROPOSED METHOD.
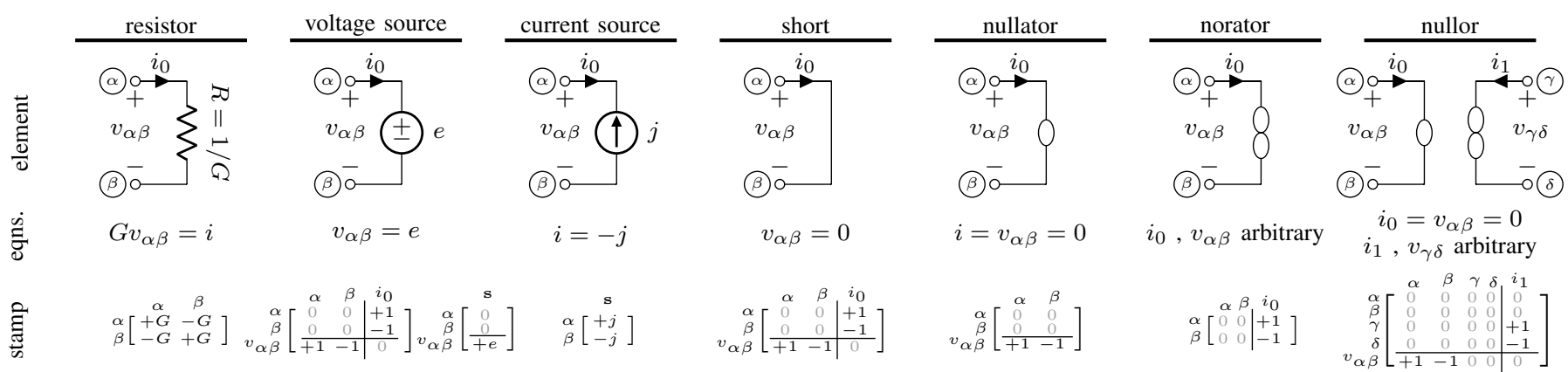

$i_{0}, v_{\alpha \beta}$ arbitrary

$$
i_{0}=v_{\alpha \beta}=0
$$

$i_{1}, v_{\gamma \delta}$ arbitrary
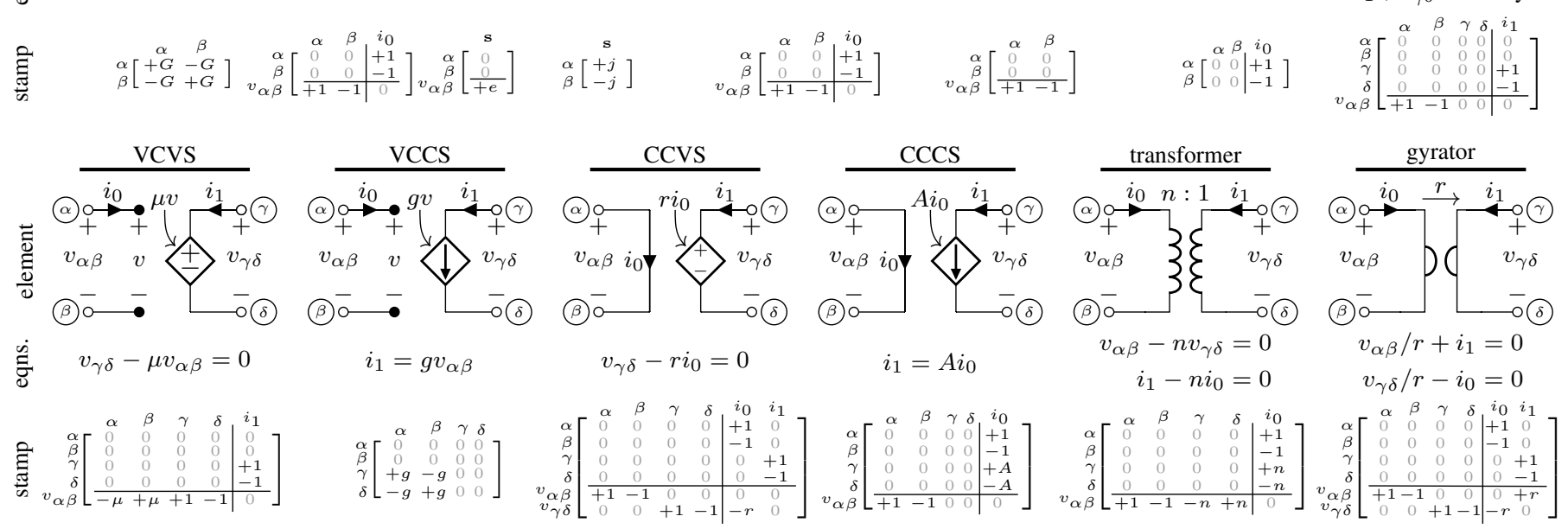

$v_{\alpha \beta} / r+i_{1}=0$

$v_{\gamma \delta} / r-i_{0}=0$
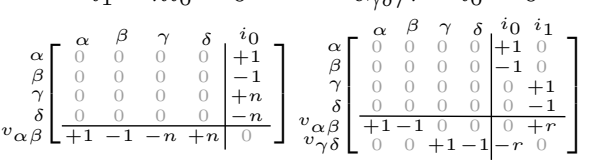

\section{Solving Circuit}

By attaching Thé./Nor. equivalents representing each incident wave to the $\mathcal{R}$-type junction, we can consider it entirely in the Kirchhoff domain. Now, the response of the junction to the incident waves can be found using a standard Kirchhoffdomain formalism: Modified Nodal Analysis (MNA) [32]. MNA sets up the equations governing a circuit in the form

$$
\begin{aligned}
& \text { KCL: } \\
& \text { extra: }
\end{aligned} \underbrace{\left[\begin{array}{ll}
\mathbf{Y} & \mathbf{B} \\
\mathbf{C} & \mathbf{D}
\end{array}\right]}_{\mathbf{X}} \underbrace{\left[\begin{array}{c}
\mathbf{v}_{\mathrm{n}} \\
\mathbf{j}_{\mathrm{b}}
\end{array}\right]}_{\mathbf{q}}=\underbrace{\left[\begin{array}{c}
\mathbf{i}_{\mathbf{s}} \\
\mathbf{e}_{\mathrm{s}}
\end{array}\right]}_{\mathbf{s}} \rightarrow \mathbf{X q}=\mathbf{s}
$$

where $\mathbf{v}_{\mathrm{n}}$ is a vector of node voltages, $\mathbf{i}_{\mathrm{s}}$ is a vector of contributions from independent current sources, and $\mathbf{e}_{\mathrm{s}}$ is a vector of independent voltage sources and zeros. The top partition represents KCL at each circuit node and $Y$ represents the admittances of electrical elements with immittance representations. The bottom partition defines other elements (e.g. voltage sources, controlled sources, nullors, etc.) that require defining extra currents $\mathbf{j}_{\mathrm{b}}$. Those extra currents influence KCL at each node through $\mathbf{B}$, and their relationship to node voltages and each other are defined through $\mathbf{C}$ and $\mathbf{D}$.

MNA systems can be written by hand, but for convenience they are usully populated using a "stamp" procedure where the influence of each electrical element is read from a table and added into the system automatically (5). Independent sources stamp into the source vector $\mathbf{s}$; the rest stamp into $\mathbf{X}$. Each "extra" element is given its own row and column in the system. Stamps, symbols, and constitutive equations for relevant oneand two-port elements are given in Tab. I [42]. In each stamp, row and column indices correspond to circuit nodes of the same name. Stamp partitions align with partitions in (5).

\section{TABLE II}

MNA AND PORT INCIDENCE STAMPS FOR THÉ. AND NOR. EQUIVALENTS.
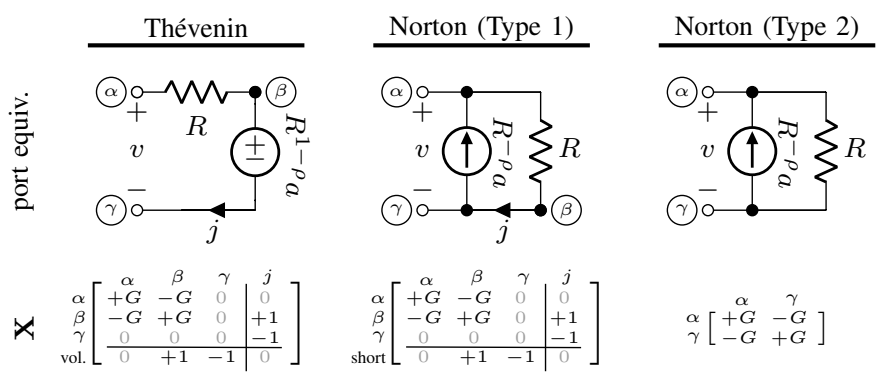

$$
\begin{array}{ll}
r_{\text {is }} & {\left[\begin{array}{cccc}
\alpha & \beta & \gamma \\
0 & 0 & 0 & j \\
r^{1-\rho_{a}}
\end{array}\right]} \\
\vdash_{4}^{a} & v_{\alpha \gamma}\left[\begin{array}{cc}
\alpha & \gamma \\
+1 & -1
\end{array}\right]
\end{array}
$$

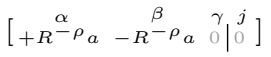$$
\left[\begin{array}{l}
-{ }^{\alpha} \rho_{a} \\
-R^{-} \rho_{a}
\end{array}\right]
$$$$
v_{\alpha \gamma}\left[\begin{array}{cc}
\alpha & \gamma \\
+1 & -1
\end{array}\right]
$$$$
v_{\alpha \gamma}\left[\begin{array}{cc}
\alpha & \gamma \\
+1 & -1
\end{array}\right]
$$

By combining multiple stamps, we create compound stamps representing Thévenin/Norton equivalents (Tab. II). We define the Thévenin stamp by combining a resistor stamp and a voltage source stamp. We define two versions of the Norton stamp: Norton Type 1 which combines resistor, current source, and short circuit stamps and Norton Type 2 which combines only a resistor stamps and current source stamp. The stamp into $\mathbf{X}$ for the Thévenin and first type of Norton source are identical. Note that the port currents are defined with respect to the junction, not to the Thévenin/Norton equivalents.

Now we partition the matrices and vectors of (5) further, defining $n+1$ as the number of nodes $(n>0)$ in the junction, $N>0$ as the number of ports, and $m \geq 0$ as the number of "extra" rows/columns. For our application, we split $\mathbf{v}_{\mathbf{n}}$ into two parts: $\mathbf{v}_{\mathrm{n}, 1} \in \mathbb{R}^{(n+1) \times 1}$ which represents nodes that are "internal" to the $\mathcal{R}$-type junction and $\mathbf{v}_{\mathrm{n}, 2} \in \mathbb{R}^{N \times 1}$ which 
represents "extra" nodes that are only part of the Thé. or Nor. (Type 1) equivalents attached to each port. At the same time $\mathbf{j}_{\mathrm{b}}$ is partitioned into two parts: $\mathbf{j}_{\mathrm{b}, 1} \in \mathbb{R}^{N \times 1}$ which represents currents (through voltage sources or shorts) involved in the Thé. or Nor. (Type 1) equivalents attached to each port and $\mathbf{j}_{\mathrm{b}, 2} \in \mathbb{R}^{m \times 1}$ which represents any extra currents needed for non-immittance multiports (controlled sources, etc.) inside of the junction. These partition the MNA system (5) as

$$
\left[\begin{array}{llll}
\mathbf{Y}_{11} & \mathbf{Y}_{12} & \mathbf{B}_{11} & \mathbf{B}_{12} \\
\mathbf{Y}_{21} & \mathbf{Y}_{22} & \mathbf{B}_{21} & \mathbf{B}_{22} \\
\mathbf{C}_{11} & \mathbf{C}_{12} & \mathbf{D}_{11} & \mathbf{D}_{12} \\
\mathbf{C}_{21} & \mathbf{C}_{22} & \mathbf{D}_{21} & \mathbf{D}_{22}
\end{array}\right]\left[\begin{array}{c}
\mathbf{v}_{\mathrm{n}, 1} \\
\mathbf{v}_{\mathrm{n}, 2} \\
\mathbf{j}_{\mathrm{b}, 1} \\
\mathbf{j}_{\mathrm{b}, 2}
\end{array}\right]=\left[\begin{array}{c}
\mathbf{i}_{\mathrm{s}, 1} \\
\mathbf{i}_{\mathrm{s}, 2} \\
\mathbf{e}_{\mathrm{s}, 1} \\
\mathbf{e}_{\mathrm{s}, 2}
\end{array}\right]
$$

partitioning $\mathbf{i}_{\mathrm{s}}$ into $\mathbf{i}_{\mathrm{s}, 1} \in \mathbb{R}^{(n+1) \times 1}$ and $\mathbf{i}_{\mathrm{s}, 2} \in \mathbb{R}^{N \times 1}$, $\mathbf{e}_{\mathrm{s}}$ into $\mathbf{e}_{\mathrm{s}, 1} \in \mathbb{R}^{N \times 1}$ and $\mathbf{e}_{\mathrm{s}, 2} \in \mathbb{R}^{m \times 1}$, and $\mathbf{Y}, \mathbf{B}, \mathbf{C}, \mathbf{D}$ into four partitions each. Since independent voltage sources only occur in Thé. equivalents, $\mathbf{e}_{\mathrm{s}, 2}=\mathbf{0}^{m \times 1}$, where $\mathbf{0}$ is a zero matrix. Since independent current sources only occur in Nor. equivalents, whose stamps only contribute currents to KCL at "internal" nodes $\mathbf{v}_{\mathrm{n}, 1}, \mathbf{i}_{\mathrm{s}, 2}=\mathbf{0}^{N \times 1}$. On account of the form of the Thé./Nor. stamps (Tab. II), $\mathbf{e}_{\mathrm{s}, 1}$ and $\mathbf{i}_{\mathrm{s}, 1}$ are factored as

$$
\mathbf{e}_{\mathrm{s}, 1}=\mathbf{R}^{1-\rho} \mathbf{a} \quad \text { (7) } \quad \mathbf{i}_{\mathrm{s}, 1}=\mathbf{A}_{\mathrm{p}} \mathbf{R}^{-\rho} \mathbf{a}
$$

where $\mathbf{A}_{\mathrm{p}} \in\{-1,0,+1\}^{(n+1) \times N}$ is a "port incidence matrix" loosely related to the incidence matrix used in the tableau formalism [42]. Each entry in $\mathbf{A}_{\mathrm{p}}$ is zero except for a single +1 and -1 in each column corresponding to the positive and negative terminals of a Thé./Nor. equivalent.

Since independent voltage sources and shorts are one-ports, $\mathbf{D}_{11}=\mathbf{0}^{N \times N}$ and $\mathbf{D}_{12}=\mathbf{D}_{21}^{\top}=\mathbf{0}^{N \times m}$. The only nonimmittance elements that can interact with the "extra" nodes $\mathbf{v}_{\mathrm{n}, 2}$ are (Thévenin) voltage sources and (Norton, Type 1) shorts contained within $\mathbf{j}_{\mathrm{b}, 1}$; therefore $\mathbf{B}_{22}=\mathbf{C}_{22}=\mathbf{0}^{N \times m}$.

The nodes $\mathbf{v}_{\mathrm{n}, 1}$ may be ordered in any fashion. When Thé. or Nor. (type 1) equivalents are used, ordering the nodes $\mathbf{v}_{\mathrm{n}, 2}$ in the same order as the ports will make $\mathbf{X}$ more orderly by causing $\mathbf{B}_{21}=\mathbf{C}_{12}=\mathbf{I}^{N \times N}$ and $\mathbf{Y}_{22}=\mathbf{G}=\mathbf{R}^{-1}$, where $\mathbf{I}$ is the identity matrix. $\mathbf{B}_{11}=\mathbf{C}_{11}^{\top} \in\{-1,0\}^{(n+1) \times N}$ is a negative permutation matrix, i.e., each column has a single -1 representing the negative terminal of an independent voltage source (Thé.) or short circuit (Nor. Type 1). $\mathbf{Y}_{11} \in$ $\mathbb{R}^{(n+1) \times(n+1)}, \mathbf{Y}_{12} \in \mathbb{R}^{(n+1) \times N}$, and $\mathbf{Y}_{21} \in \mathbb{R}^{N \times(n+1)}$, and $\mathbf{Y}_{22} \in \mathbb{R}^{N \times N}$ encode admittance relationships between node voltages. $\mathbf{B}_{12} \in \mathbb{R}^{(n+1) \times m}, \mathbf{C}_{21} \in \mathbb{R}^{m \times m}$, and $\mathbf{D}_{22} \in \mathbb{R}^{m \times m}$ encode relationships imposed by multiport elements (except VCCSs, which contribute to $\mathbf{Y}_{11}$ ) inside the junction.

There are two particular forms of $\mathbf{X}$, one $\left(\mathbf{X}_{0}\right)$ for the case that Nor. equivalents (Type 2) are used to represent incident waves and node voltages are used to solve the scattering relationship and one $\left(\mathbf{X}_{1}\right)$ for the other three cases

$$
\mathbf{X}_{0}=\left[\begin{array}{ll}
\mathbf{Y}_{11} & \mathbf{B}_{12} \\
\mathbf{C}_{21} & \mathbf{D}_{22}
\end{array}\right], \quad \mathbf{X}_{1}=\left[\begin{array}{cccc}
\mathbf{Y}_{11} & \mathbf{Y}_{12} & \mathbf{B}_{11} & \mathbf{B}_{12} \\
\mathbf{Y}_{21} & \mathbf{G} & \mathbf{I} & \mathbf{0} \\
\mathbf{C}_{11} & \mathbf{I} & \mathbf{0} & \mathbf{0} \\
\mathbf{C}_{21} & \mathbf{0} & \mathbf{0} & \mathbf{D}_{22}
\end{array}\right] .
$$

Twelve partitions $\left(\mathbf{Y}_{12}, \mathbf{Y}_{21}, \mathbf{Y}_{22}, \mathbf{B}_{11}, \mathbf{B}_{21}, \mathbf{B}_{22}, \mathbf{C}_{11}, \mathbf{C}_{12}\right.$, $\mathbf{C}_{22}, \mathbf{D}_{11}, \mathbf{D}_{12}$, and $\mathbf{D}_{21}$ ) are missing from $\mathbf{X}_{0}$ because, for Nor. Type 2 equivalents, there are no "external nodes" $\left(\mathbf{v}_{n, 2}\right.$ and $\mathbf{i}_{\mathrm{s}, 2}$ do not exist) and there are no explicit shorts or Thé. currents $\left(\mathbf{j}_{\mathrm{b}, 1}\right.$ and $\mathbf{e}_{\mathrm{s}, 1}$ do not exist).
Likewise, we have three different forms of the source vector $\mathbf{s}$, one $\left(\mathbf{s}_{1}\right)$ when Thévenin equivalents are used, one $\left(\mathbf{s}_{2}\right)$ for Norton Type 1 , and one $\left(\mathbf{s}_{0}\right)$ for Norton Type 2

$$
\begin{aligned}
& \text { Cur./Vol.+Thé. : } \quad \mathbf{s}_{1}=\left[\begin{array}{llll}
\mathbf{0} & \mathbf{0} & \mathbf{I} & \mathbf{0}
\end{array}\right]^{\top} \mathbf{R}^{1-\rho} \mathbf{a} \\
& \text { Cur.+Nor. : } \quad \mathbf{s}_{2}=\left[\begin{array}{llll}
\mathbf{I} & \mathbf{0} & \mathbf{0} & \mathbf{0}
\end{array}\right]^{\top} \mathbf{A}_{\mathrm{p}} \mathbf{R}^{-\rho} \mathbf{a} \\
& \text { Vol.+Nor. : } \quad \mathbf{s}_{0}=\left[\begin{array}{ll}
\mathbf{I} & \mathbf{0}
\end{array}\right]^{\top} \mathbf{A}_{\mathrm{p}} \mathbf{R}^{-\rho} \mathbf{a} \text {. }
\end{aligned}
$$

The MNA system (6) needs to be solved by inverting the matrix $\mathbf{X}$. However by construction $\mathbf{X}$ is not full rank since (6) includes KCL equations for all $n+1$ node voltages, but circuits have only $n$ independent KCL constraints [42]. This is resolved by removing the influence of one circuit node (it does not matter which one) called the "datum node" $d$ from all equations. This essentially sets $v_{d}=0$, or treats it as ground. Put another way, it solves all other node voltages with respect to $v_{d}$. Although the actual ground node is often set as the datum node, the circuit's ground node will not necessarily be part of any particular $\mathcal{R}$-type topology. For notational reasons, we require removing an "internal" node, whose voltage $v_{d} \in \mathbf{v}_{\mathrm{n}, 1}$. Mathematically, removing the datum node removes a node voltage from $\mathbf{v}_{\mathrm{n}, 1}$, ignores the influence of current sources that would contribute to KCL at that node, and removes a row and column from $\mathbf{X}$. This operation on $\mathbf{X}$ amounts to removing a row and column from $\mathbf{Y}_{11}$; a row from $\mathbf{Y}_{12}, \mathbf{B}_{11}$, and $\mathbf{B}_{12}$; and a column from $\mathbf{Y}_{21}, \mathbf{C}_{11}$, and $\mathbf{C}_{21}$. The incidence matrix $\mathbf{A}_{\mathrm{p}}$ also must have the appropriate row removed. Versions of variables with the influence of the datum node removed are indicated with a tilde overhead, e.g. $\tilde{\mathbf{v}}_{\mathrm{n}, 1}$ or $\tilde{\mathbf{A}}_{\mathrm{p}}$. The datum-node-less system is solved by $\tilde{\mathbf{q}}=\tilde{\mathbf{X}}^{-1} \tilde{\mathbf{s}}$.

\section{E. Forming Outputs}

Recall from (2) that $\mathbf{b}$ is a linear combination of port voltages and currents. Knowing $\mathbf{q}$ allows us to find $\mathbf{b}$ by eliminating either $\mathbf{v}$ or $\mathbf{i}$ in (2)

$$
\mathbf{b}=\mathbf{a}-2 \mathbf{R}^{\rho} \mathbf{i} \quad \text { (13) } \quad \mathbf{b}=2 \mathbf{R}^{\rho-1} \mathbf{v}-\mathbf{a}
$$

leaving the task of finding $\mathbf{i}$ or $\mathbf{v}$ from $\tilde{\mathbf{q}}$. $\mathbf{i}$ can be found from $\mathbf{j}_{\mathrm{b}, 1}$ which represents the currents through Thévenin voltage sources or Norton (Type 1) short circuits. $\mathbf{v}$ can be found from $\mathbf{v}_{\mathrm{n}}$ using the port incidence matrix $\tilde{\mathbf{A}}_{\mathrm{p}}$ :

$$
\begin{array}{ll}
\text { Cur.+Thé. } & : \quad \mathbf{i}=-\mathbf{j}_{\mathrm{b}, 1} \\
\text { Cur.+Nor. } & : \quad \mathbf{i}=\mathbf{R}^{-\rho} \mathbf{a}-\mathbf{j}_{\mathrm{b}, 1} \\
\text { Vol.+Thé./Nor. }: & \mathbf{v}=\tilde{\mathbf{A}}_{\mathrm{p}} \tilde{\mathbf{v}}_{\mathrm{n}, 1} .
\end{array}
$$

The current vectors $\mathbf{j}_{\mathrm{b}, 1}$ or node voltage vector $\mathbf{v}_{\mathrm{n}}$ are found simply by selecting the appropriate entries from $\tilde{\mathbf{q}}$

$$
\begin{aligned}
& \text { Cur.+Thé./Nor. : } \mathbf{j}_{\mathrm{b}, 1}=\left[\begin{array}{llll}
\mathbf{0} & 0 & \mathbf{I} & \mathbf{0}
\end{array}\right] \tilde{\mathbf{q}} \\
& \text { Vol.+Thé./Nor. : } \quad \tilde{\mathbf{v}}_{\mathrm{n}}=\left[\begin{array}{llll}
\mathbf{I} & 0 & 0 & 0
\end{array}\right] \tilde{\mathbf{q}} \text {. }
\end{aligned}
$$

\section{F. Scattering Matrices}

Combining the two options for representing incident waves (Thé./Nor. equivalent circuits, §II-C) with the two options for forming reflected waves ((13) with (15) or (16), and (18); or 
TABLE III

SCATTERING FOR EACH COMBINATION OF PORT EQUIVALENT AND SOLUTION BASES PLUS VECTOR AND MATRIX SIZES AND COST IN MULTIPLIES.

\begin{tabular}{|c|c|c|c|c|c|c|c|}
\hline \multicolumn{2}{|c|}{ bases } & & \multirow[b]{2}{*}{ eqn. } & \multirow[b]{2}{*}{ fixed cost } & \multicolumn{3}{|c|}{ cost, $\rho=$} \\
\hline solve & equivalent & scattering equation & & & 1 & 0 & else \\
\hline Current & Thévenin & 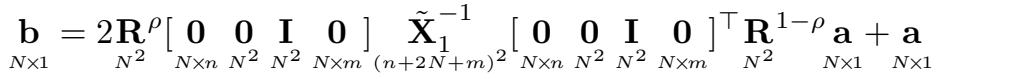 & (24) & $N^{2}$ & $N$ & $N$ & $2 N$ \\
\hline Current & Norton & 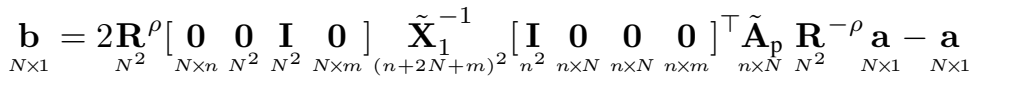 & $(25)$ & $N \times n$ & 0 & $2 N$ & $2 N$ \\
\hline Voltage & Thévenin & 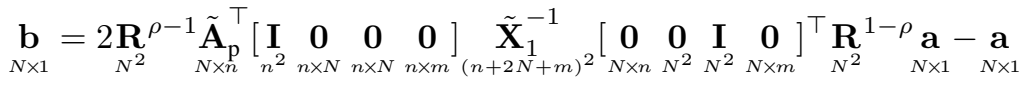 & $(26)$ & $N \times n$ & $2 N$ & 0 & $2 N$ \\
\hline Voltage & Norton & 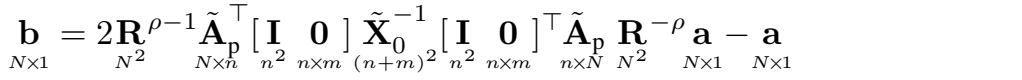 & (27) & $n^{2}$ & $N$ & $N$ & $2 N$ \\
\hline
\end{tabular}

(14), (17), and (19) ) yields four different equations for the scattering matrix $\mathbf{S} \in \mathbb{R}^{N \times N}$ of an $\mathcal{R}$-type junction:

$$
\begin{aligned}
& \mathbf{S}=2 \mathbf{R}^{\rho}\left[\begin{array}{llll}
\mathbf{0} & \mathbf{0} & \mathbf{I} & \mathbf{0}
\end{array}\right] \tilde{\mathbf{X}}_{1}^{-1}\left[\begin{array}{llll}
\mathbf{0} & \mathbf{0} & \mathbf{I} & \mathbf{0}
\end{array}\right]^{\top} \mathbf{R}^{1-\rho}+\mathbf{I} \\
& \mathbf{S}=2 \mathbf{R}^{\rho}\left[\begin{array}{llll}
\mathbf{0} & \mathbf{0} & \mathbf{I} & \mathbf{0}
\end{array}\right] \tilde{\mathbf{X}}_{1}^{-1}\left[\begin{array}{llll}
\mathbf{I} & \mathbf{0} & \mathbf{0} & \mathbf{0}
\end{array}\right]^{\top} \tilde{\mathbf{A}}_{\mathrm{p}} \mathbf{R}^{-\rho}-\mathbf{I} \\
& \mathbf{S}=2 \mathbf{R}^{\rho-1} \tilde{\mathbf{A}}_{\mathbf{p}}^{\top}\left[\begin{array}{lllll}
\mathbf{I} & \mathbf{0} & \mathbf{0} & \mathbf{0}
\end{array}\right] \tilde{\mathbf{X}}_{1}^{-1}\left[\begin{array}{llll}
\mathbf{0} & \mathbf{0} & \mathbf{I} & \mathbf{0}
\end{array}\right]^{\top} \mathbf{R}^{1-\rho}-\mathbf{I} \\
& \mathbf{S}=2 \mathbf{R}^{\rho-1} \tilde{\mathbf{A}}_{\mathbf{p}}^{\top}\left[\begin{array}{lll}
\mathbf{I} & \mathbf{0}
\end{array}\right] \tilde{\mathbf{X}}_{0}^{-1}\left[\begin{array}{lll}
\mathbf{I} & \mathbf{0}
\end{array}\right]^{\top} \tilde{\mathbf{A}}_{\mathrm{p}} \mathbf{R}^{-\rho}-\mathbf{I}
\end{aligned}
$$

where $\mathbf{S}$ solves the relationship $\mathbf{b}=\mathbf{S a}$. The first, (20) is a generalization of the equation seen previously, e.g. in [25].

$\mathcal{R}$-type adaptors with scattering matrix $\mathbf{S}$ are suitable for use in WDF CTs where, as with other adaptors, they have one parent above and $N-1$ children below. To create a computable structure, the upwards-facing port must be rendered reflectionfree ("adapted") by choosing its port resistance so that the corresponding diagonal entry of $\mathbf{S}$ is set to zero.

\section{G. Alternative Implementation of Scattering}

Calculating $\mathbf{b}=\mathbf{S a}$ might be expensive since it costs $N^{2}$ multiplies. However, in order to compute $\mathbf{b}$, forming $\mathbf{S}$ is not actually necessary (similar to [43]). Instead, (20)-(23) and the scattering definition are combined to form (24)-(27). Tab. III shows each equation, its solution bases (port Currents/Voltages and Thé./Nor. equivalents), the size of each matrix and vector, and the upper bound on its cost in multiplies.

"Cost" here means the number of multiplications needed to implement a scattering operation assuming that all matrices have been computed ahead of time. It is calculated by adding the "fixed cost" column from Tab. III to the appropriate column from "cost, $\rho=$ " also from Tab. III. The $\rho$-dependent cost comes from two diagonal-matrix-vector multiplications by $\mathbf{R}$ raised to a power. Since $\mathbf{R}^{0}=\mathbf{I}$, one or both of these diagonalmatrix-vector multiplies sometimes has no cost, depending on $\rho$. The fixed cost comes from a matrix-vector multiplication by $\tilde{\mathbf{X}}_{0}$ or $\tilde{\mathbf{X}}_{1}$ surrounded by matrixes partitioned into $\mathbf{0}$ and I matrices. This cost is the product of the dimensions of the two I matrices. Since $\tilde{\mathbf{A}}_{\mathrm{p}} \in\{-1,0,+1\}$, multiplications by $\tilde{\mathbf{A}}_{\mathrm{p}}$ involve some sign inversions, but no scalar multiplications. The leading scalar, 2, can be embedded into one of the $\mathbf{R}$ or $\tilde{\mathbf{X}}$ matrices, so it does not contribute any additional multiplies.

For (24)-(27), costs less than $N^{2}$ should be considered; otherwise the scattering should be implemented in the standard fashion. We discard (24), whose cost is greater than $N^{2}$ by construction, retaining (25)-(27) which will in many cases cost less than $N^{2}$. Typically, $n<N$, so (27) will typically be the most economical implementation of the scattering relationship.

Analyzing the computational cost of these scattering operations for time-varying circuits where some matrices may need to be recomputed on the fly is beyond the scope of this article. However, we briefly mention that $\tilde{\mathbf{X}}_{0}$ is smaller than $\tilde{\mathbf{X}}_{1}$ by construction and should be cheaper to invert, potentially leading to a preference for (27) for time-varying circuits.

\section{Structures With Multiple Wave Types}

When active junctions present a negative incremental resistance [23], [44] at their upwards-facing port, the WDF adaptor of that junction will need to be adapted by a negative port resistance. In this case, values of $\rho$ other than 1 or 0 in the $\mathbf{R}$ exponents of (20)-(23) or (24)-(27) cause complex scattering coefficients and signal values that are not physically meaningful in a transient simulation, restricting the simulation to voltage and current waves. We propose two different remedies that localize this restriction to only a portion of the simulation.

\section{A. Wave Converter Two-Port}

A first approach involves defining a new WDF two-port (Fig. 3) that interfaces between two WDF simulations with different wave definitions $\left(\rho_{1}\right.$ and $\left.\rho_{2}\right)$. We start with a simple port connection (i.e., a two-port parallel connection, Fig. 3a):

$$
v_{1}=v_{2} \quad \text { (28) } \quad i_{1}=-i_{2}
$$

Plugging the parametric wave definition (1) into (28)-(29), collecting the results into a matrix equation, and solving for $\left[\begin{array}{ll}b_{1} & b_{2}\end{array}\right]^{\top}$ yields the scattering relationship

$$
\left[\begin{array}{l}
b_{1} \\
b_{2}
\end{array}\right]=\left[\begin{array}{cc}
-\frac{R_{1}-R_{2}}{R_{1}+R_{2}} & \frac{2 R_{1}^{\rho_{1}} R_{2}^{1-\rho_{2}}}{R_{1}+R_{2}} \\
\frac{2 R_{1}^{1-\rho_{1}} R_{2}^{\rho_{2}}}{R_{1}+R_{2}} & \frac{R_{1}-R_{2}}{R_{1}+R_{2}}
\end{array}\right]\left[\begin{array}{l}
a_{1} \\
a_{2}
\end{array}\right]
$$

whose signal flow is shown in Fig. 3c. The upwards-facing port (port 1) of this wave converter can be adapted by choosing its port resistance $R_{1}$ to zero out the diagonal entries of the scattering matrix. Setting $R_{1}=R_{2}$, yields

$$
\left[\begin{array}{l}
b_{1} \\
b_{2}
\end{array}\right]=\left[\begin{array}{cc}
0 & R_{2}^{\rho_{1}-\rho_{2}} \\
R_{2}^{\rho_{2}-\rho_{1}} & 0
\end{array}\right]\left[\begin{array}{l}
a_{1} \\
a_{2}
\end{array}\right],
$$




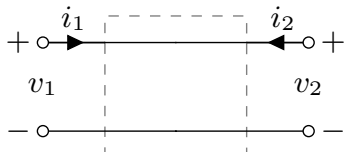

(a) Kirchhoff domain.

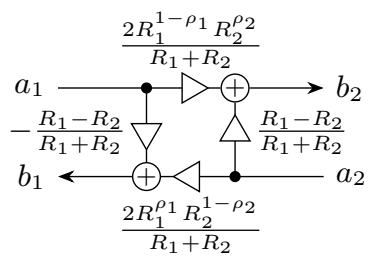

(c) Unadapted signal flow.

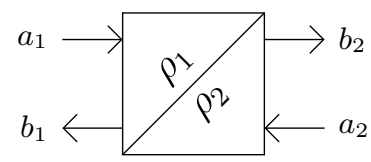

(b) WDF block.

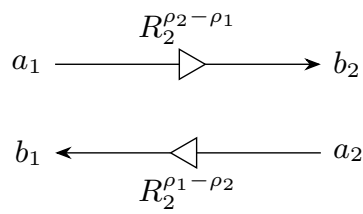

(d) Adapted signal flow.
Fig. 3. Two-port wave converter definition.

whose signal flow is shown in Fig. $3 \mathrm{~d}$. When $\rho_{1}=\rho_{2}$ this is a standard parallel connection between ports.

An example application of the wave converter two-port is shown in the Frequency Booster case study ( $\mathrm{IV}-\mathrm{B})$.

\section{B. Hybrid-Wave Junction}

Wave converter two-ports can interface parts of a WDF with different parametric wave definitions. Here we propose an alternate approach that allows multiple wave types to coexist within one adaptor. We now assume that each port $k \in 1 \cdots N$ in an adaptor is parametrized by an independent wave parameter $\rho_{k}$, i.e., the $N$-port adaptor is parametrized by a vector $\boldsymbol{\rho}=\left[\rho_{1}, \rho_{2}, \cdots, \rho_{N}\right]^{\top}$. The scattering matrix equations (20)-(23) or scattering implementations (24)-(27) are affected in the following way. Each port resistance matrix raised to a power $\left(\mathbf{R}^{x}\right)$ is replaced by an "diagonal elementwise power" $\mathbf{R}^{(\mathbf{x})}=\operatorname{diag}\left(\left[R_{1}^{x_{1}}, R_{2}^{x_{2}}, \cdots, R_{N}^{x_{N}}\right]\right)$, giving

$$
\begin{aligned}
\mathbf{R}^{\rho} & \mapsto \mathbf{R}^{(\boldsymbol{\rho})}=\operatorname{diag}\left(\left[R_{1}^{\rho_{1}}, R_{2}^{\rho_{2}}, \cdots, R_{N}^{\rho_{N}}\right]\right) \\
\mathbf{R}^{1-\rho} & \mapsto \mathbf{R}^{(1-\boldsymbol{\rho})}=\operatorname{diag}\left(\left[R_{1}^{1-\rho_{1}}, R_{2}^{1-\rho_{2}}, \cdots, R_{N}^{1-\rho_{N}}\right]\right) \\
\mathbf{R}^{\rho-1} & \mapsto \mathbf{R}^{(\boldsymbol{\rho}-1)}=\operatorname{diag}\left(\left[R_{1}^{\rho_{1}-1}, R_{2}^{\rho_{2}-1}, \cdots, R_{N}^{\rho_{N}-1}\right]\right) \\
\mathbf{R}^{-\rho} & \mapsto \mathbf{R}^{(-\boldsymbol{\rho})}=\operatorname{diag}\left(\left[R_{1}^{-\rho_{1}}, R_{2}^{-\rho_{2}}, \cdots, R_{N}^{-\rho_{N}}\right]\right)
\end{aligned}
$$

Note that all of these replacements relate to source scaling in representing incident waves by Thé./Nor. equivalents, or to the formation of reflected waves. The resistance terms that show up in $\tilde{\mathbf{X}}_{0}$ and $\tilde{\mathbf{X}}_{1}$ relate to the Thévenin or Norton resistances of the equivalents; since this does not depend on the wave parametrization these values do not need to be altered.

As an example, this approach is useful when an active $\mathcal{R}$-type adaptor's upwards-facing port must be adapted by a negative port resistance but the algorithm designer prefers power waves. The $\mathcal{R}$-type adaptor's upwards-facing port can be defined with, e.g., voltage waves $(\rho=1)$, and its other ports (as well as everything below it) can be defined with power waves $(\rho=1 / 2)$. This localizes the prohibition on power waves to only a single port and possibly other WDF elements between that port and the root of the tree.

An example application of a hybrid-wave $\mathcal{R}$-type adaptor is shown in the Frequency Booster case study (§IV-B).

\section{CAse Studies}

Here, we apply the proposed method to case studies on two audio circuits: the classic Baxandall tone control and a "Frequency Booster" guitar pedal. In each case we detail how to form a graph from the reference circuit, perform a search for split components on the graph to form an SPQR tree, and derive a WDF adaptor structure isomorphic to the SPQR tree. Revealing the internals of each adaptor makes the relationship between the WDF structures and the reference circuits clear. This analysis reveals an $\mathcal{R}$-type topology in the WDF structure of each case study. In each, the scattering matrix for the $\mathcal{R}$ type adaptor is derived using the proposed method ( $(\mathrm{II})$ and adapted by choosing the correct port resistance for its upwardsfacing port. This yields computable WDF simulations which closely match the behavior of their analog reference circuits. In all cases a sampling rate of $48 \mathrm{kHz}$ is used.

\section{A. Baxandall Tone Control}

The Baxandall tone control [45] is an early parametric audio filter that allows continuous control of bass and treble levels [46] and is common in hi-fi receivers, guitar amps, and other audio effects [47], [48]. The schematic of a version (adding a large load resistor $R_{\mathrm{L}}$ to [49, p. 2-49]) is shown in Fig. 4a. The input is an ideal voltage source $v_{\text {in }}$ and the output is the voltage across $R_{\mathrm{L}}$. The circuit comprises 5 capacitors $\left(C_{\mathrm{a}} \cdots C_{\mathrm{e}}\right), 6$ resistors $\left(R_{\mathrm{a}} \cdots R_{\mathrm{e}}\right.$ and $\left.R_{\mathrm{L}}\right)$, and two centertapped potentiometers $P_{\mathrm{B}}$ (parametrized by a bass control $B$ ) and $P_{\mathrm{T}}$ (parametrized by a treble control $T$ ). Fig. 4a shows how the potentiometers may be further broken down into pairs of resistors $P_{\mathrm{B}}^{+}=P_{\mathrm{B}, \max }(B)$ and $P_{\mathrm{B}}^{-}=P_{\mathrm{B}, \max }(1-B)$, respectively $P_{\mathrm{T}}^{+}=P_{\mathrm{T}, \max }(T)$ and $P_{\mathrm{T}}^{-}=P_{\mathrm{T}, \max }(1-T)$.

1) WDF Model: A graph representing the circuit is shown in Fig. 4c. Circuit nodes correspond to graph nodes a $\cdots \mathrm{i}$ and electrical components correspond to graph edges $1 \cdots 16$; component values and mappings to graph edges are given in Fig. 4b. Using the graph separation algorithm of [26] yields a decomposition into 5 series $\left(\mathcal{S}_{1} \cdots \mathcal{S}_{5}\right), 5$ parallel $\left(\mathcal{P}_{1} \cdots \mathcal{P}_{5}\right)$, and 1 rigid connection $\left(\mathcal{R}_{1}\right)$. Using the notation of [26], this is shown in Fig. 4d. Designating $v_{\text {in }}$ (edge 1) as the root, the graph decomposition yields an SPQR tree (Fig. 4e) isomorphic to a WDF adaptor structure (Fig. 4f) retaining the topology of the reference circuit (Fig. 4g). The series/parallel adaptors and one-ports are implemented using classical WDF techniques, the capacitors are discretized using the bilinear transform [1], and power waves $(\rho=1 / 2)$ are used. $\mathcal{R}_{1}$ 's scattering is implemented using the proposed method. As with other WDF adaptors, its upward-facing (towards $\mathcal{S}_{1}$ ) port resistance is chosen to make that port reflection-free. By setting

$$
R_{\mathrm{F}}=\frac{\left(R_{\mathrm{A}}\left(R_{\mathrm{B}}+R_{\mathrm{C}}\right)+R_{\mathrm{B}} R_{\mathrm{C}}\right)\left(R_{\mathrm{D}}+R_{\mathrm{E}}\right)+\left(R_{\mathrm{A}}+R_{\mathrm{B}}\right) R_{\mathrm{D}} R_{\mathrm{E}}}{R_{\mathrm{A}}\left(R_{\mathrm{B}}+R_{\mathrm{C}}+R_{\mathrm{D}}\right)+R_{\mathrm{B}}\left(R_{\mathrm{C}}+R_{\mathrm{E}}\right)+R_{\mathrm{C}}\left(R_{\mathrm{D}}+R_{\mathrm{E}}\right)+R_{\mathrm{D}} R_{\mathrm{E}}}
$$

port $\mathrm{F}$ is adapted $\left(\mathbf{S}_{\mathrm{FF}}\right.$ set to 0$)$.

2) Discussion: The model is characterized for a variety of bass and treble control settings $B, T \in] 0,1[$ in Fig. 5. As a practical measure, $B$ and $T$ cannot be set all the way to their extremal values, since this would cause port resistances of zero (a non-invertible wave definition) in the WDF model. 


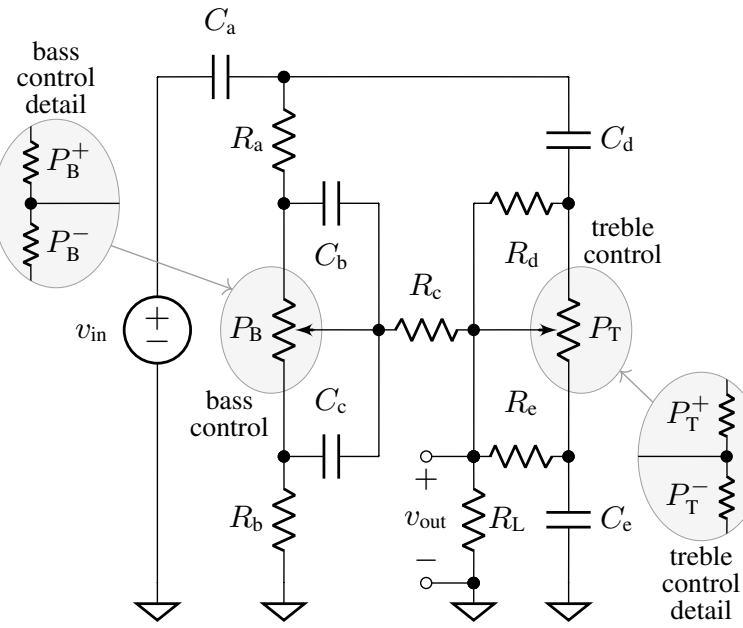

(a) Reference Circuit.

\begin{tabular}{lll}
\hline Value & Comp. & Edge \\
\hline input signal & $v_{\text {in }}$ & 1 \\
$10 \mathrm{k} \Omega$ & $R_{\mathrm{a}}$ & 3 \\
$1 \mathrm{k} \Omega$ & $R_{\mathrm{b}}$ & 8 \\
$10 \mathrm{k} \Omega$ & $R_{\mathrm{c}}$ & 9 \\
$10 \mathrm{k} \Omega$ & $R_{\mathrm{d}}$ & 11 \\
$1 \mathrm{k} \Omega$ & $R_{\mathrm{e}}$ & 13 \\
$1 \mathrm{M} \Omega$ & $R_{\mathrm{L}}$ & 16 \\
$100 \mathrm{k} \Omega$ & $P_{\mathrm{B}, \max }$ & - \\
$P_{\mathrm{B}, \max }(B)$ & $P_{\mathrm{B}}^{+}$ & 4 \\
$P_{\mathrm{B}, \max }(1-B)$ & $P_{\mathrm{B}}^{-}$ & 6 \\
$100 \mathrm{k} \Omega$ & $P_{\mathrm{T}}, \max$ & \\
$P_{\mathrm{T}, \max }(T)$ & $P_{\mathrm{T}}^{+}$ & 12 \\
$P_{\mathrm{T}, \max }(1-T)$ & $P_{\mathrm{T}}^{-}$ & 14 \\
$1 \mu \mathrm{F}$ & $C_{\mathrm{a}}$ & 2 \\
$33 \mathrm{nF}$ & $C_{\mathrm{b}}$ & 5 \\
$330 \mathrm{nF}$ & $C_{\mathrm{c}}$ & 7 \\
$15 \mathrm{nF}$ & $C_{\mathrm{d}}$ & 10 \\
$150 \mathrm{nF}$ & $C_{\mathrm{e}}$ & 15 \\
\hline
\end{tabular}

(b) Components to Graph Edges.

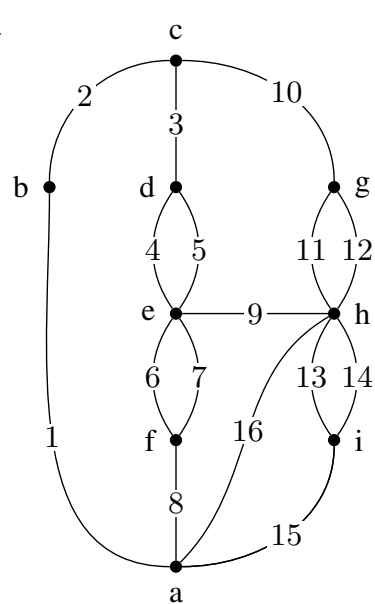

(c) Reference Circuit Graph.

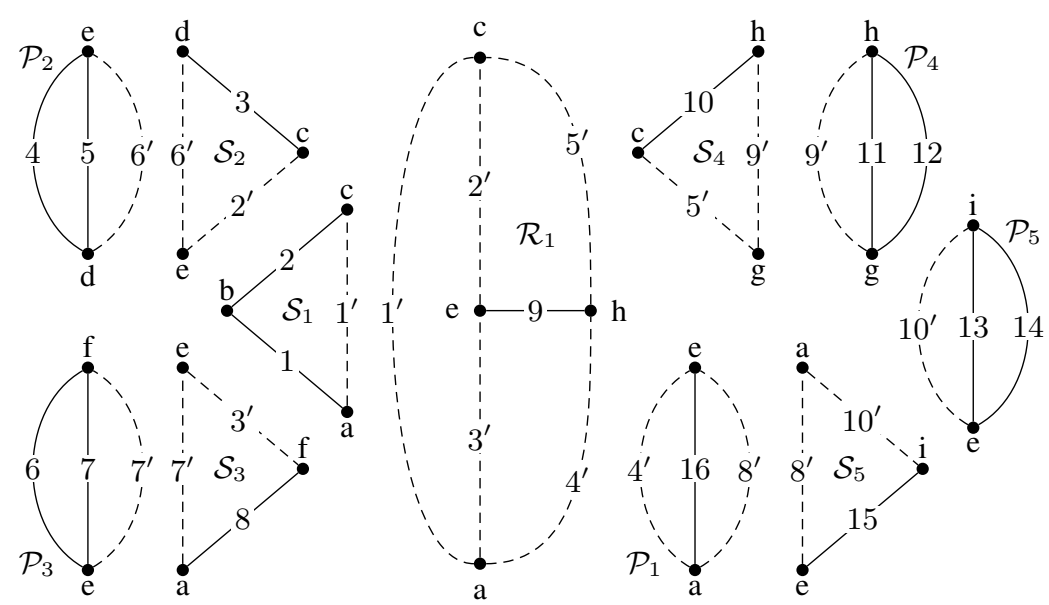

(d) Reference Circuit Graph Separated.

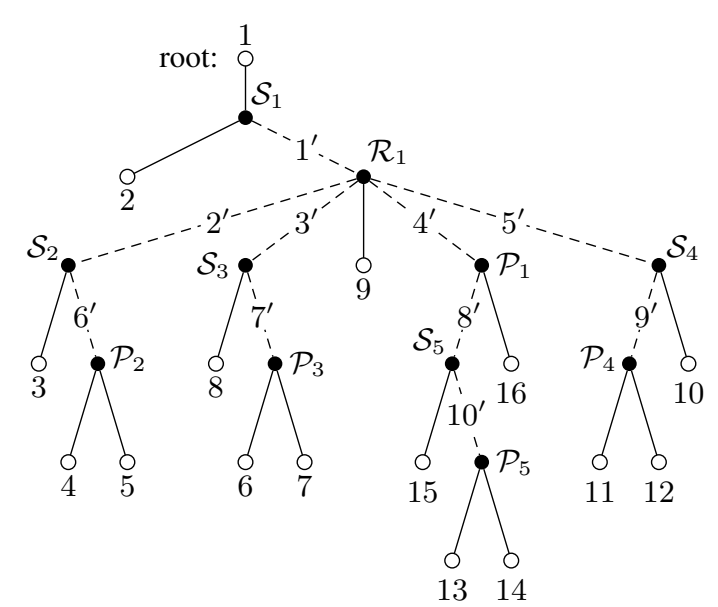

(e) SPQR Connection Tree (CT).

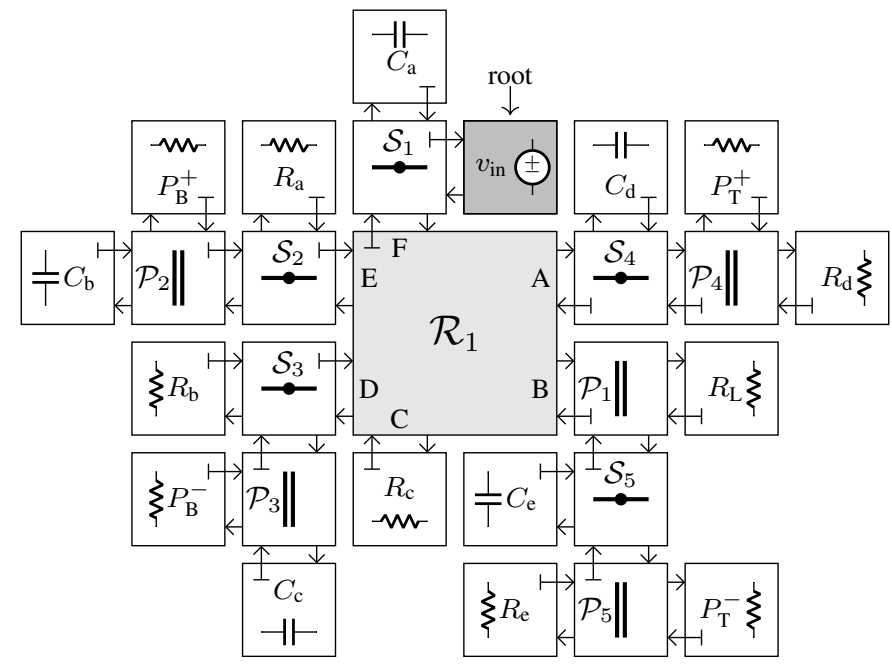

(f) Wave Digital Filter.

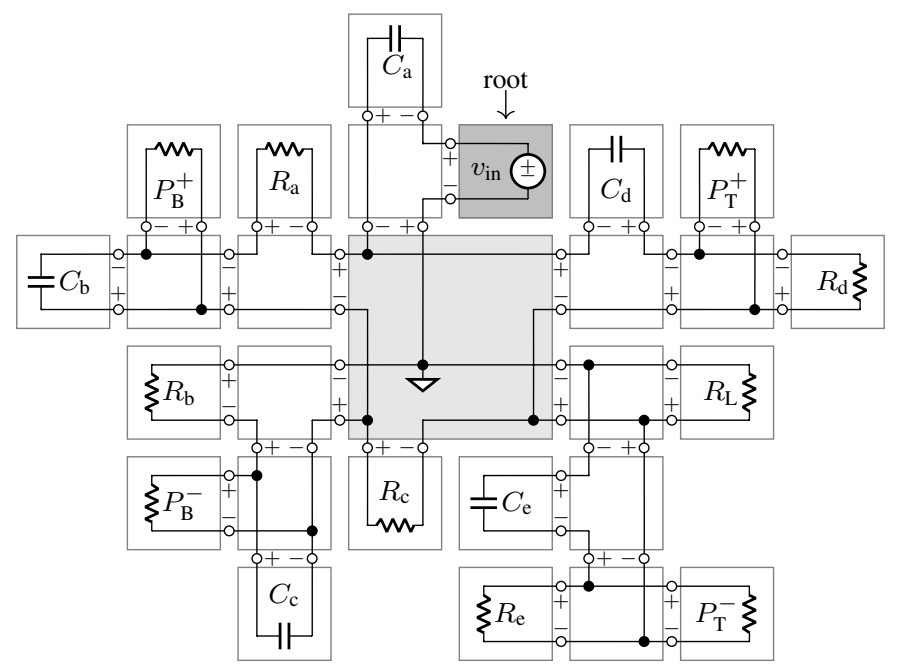

(g) Reference Circuit Rearranged.

Fig. 4. Steps for deriving Baxandall tone control WDF simulation from schematic, including graph representation, graph separation, and SPQR steps. Treble control setting $T \in] 0,1[$. Bass control setting $B \in] 0,1[$. 


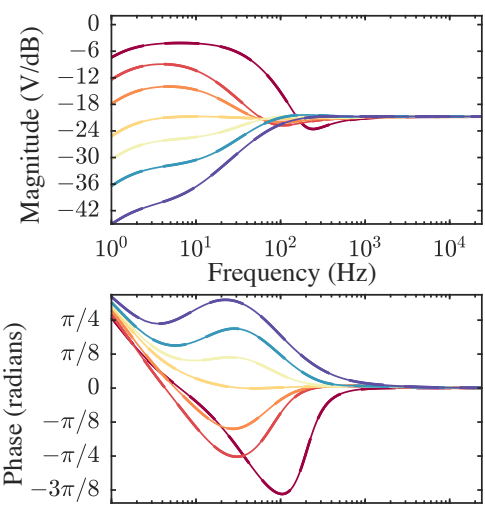

(a) Varying bass control $B$.
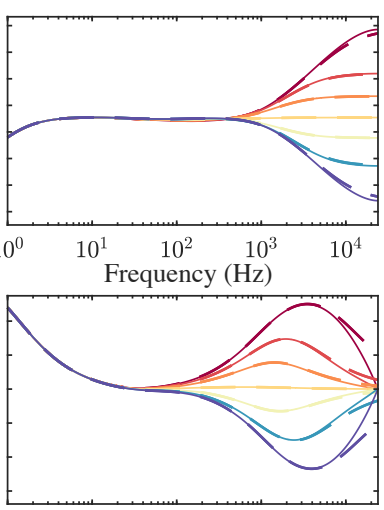

(b) Varying treble control $T$.
Fig. 5. Magnitude $|H|$ and phase $\angle H$ responses of Baxandall tone control.

TABLE IV

QUANTITIES, CASE STUDY $\mathcal{R}$-TYPE ADAPTOR IMPLEMENTATIONS

\begin{tabular}{lccc}
\hline Number of & variable & Baxandall tone & Frequency Booster \\
\hline Ports & $N$ & 6 & 8 \\
(Non-datum) nodes & $n$ & 3 & 5 \\
Extra MNA variables & $m$ & 0 & 1 \\
\hline
\end{tabular}

Figs. 5a-5b show frequency responses $H=V_{\text {out }} / V_{\text {in }}$ for various bass control settings $B \in] 0,1[$ (treble control fixed at $T=0.1$ ) and various treble control settings $T \in] 0,1[$ (bass control fixed at $B=0.1$ ). The WDF model's frequency responses are found by taking the Discrete Fourier Transform of measured unit-amplitude impulse responses. Dashed lines showing the analog response calculated using SPICE [21] and solid lines showing the WDF response have excellent agreement. The only visible distortions are the expected highfrequency warping from the bilinear transform [50]. The Baxandall tone control has weakly parametric controls. For instance, the bass control $B$ primarily affects a bass "shelf" level but also affects the cutoff frequency, ripple in the transition band, shape of the shelf corners, etc. Control parametrization is an important part of VA [51]; by modeling the circuit using WDFs we obtain this parametrization as a byproduct of properly modeling the reference circuit's dynamics.

Relevant quantities for analyzing $\mathcal{R}_{1}$ 's implementation cost in multiplies are shown in Tab. IV. The costs of (24)-(27) (from Tab. III) are shown for various wave types and compared against nominal scattering matrix multiplication (20)-(23) and the method of Martens and Meerkötter [27] in Tab. V. The minimum implementation cost for each wave type is darkly highlighted and bolded, and any implementation that is cheaper than the nominal is lightly highlighted. For $\rho \neq 1$, using (27) (Nor.+Vol.) minimizes the number of multiplies, reducing the cost compared to the nominal by about $58.3 \%$ or $41.7 \%$, depending on the wave type. For voltage waves $(\rho=$ 1), Martens and Meerkötter has the lowest implementation cost. This is the case in general for voltage-wave simulations of passive reciprocal circuits. However, while the method of Martens and Meerkötter cannot be used at all for other wave types, our proposed approach suffers no such restrictions.
TABLE V

NO. OF MULTIPLIES, CASE STUDY $\mathcal{R}$-TYPE ADAPTOR IMPLEMENTATIONS

\begin{tabular}{|c|c|c|c|c|c|c|c|c|c|}
\hline \multirow[b]{3}{*}{ eq. } & & & \multicolumn{3}{|c|}{ Baxandall tone } & \multicolumn{4}{|c|}{ Frequency Booster } \\
\hline & \multicolumn{2}{|c|}{ basis } & \multicolumn{3}{|c|}{$\rho=$} & \multicolumn{4}{|c|}{$\rho=$} \\
\hline & sol. & equiv. & 1 & 0 & else & 1 & 0 & else & hybrid \\
\hline (24) & Cur. & Thé. & 42 & 42 & 48 & 72 & 72 & 80 & 79 \\
\hline (25) & Cur. & Nor. & 30 & 18 & 30 & 56 & 40 & 56 & 54 \\
\hline (26) & Vol. & Thé. & 18 & 30 & 30 & 40 & 56 & 56 & 56 \\
\hline (27) & Vol. & Nor. & 15 & 15 & 21 & 33 & 33 & 41 & 40 \\
\hline (20)-(23) & \multicolumn{2}{|c|}{ nominal $\mathbf{S}$} & 36 & 36 & 36 & 64 & 64 & 64 & 64 \\
\hline \multicolumn{3}{|c|}{ Martens \& Meerkötter } & 9 & - & - & - & - & - & - \\
\hline
\end{tabular}

\section{B. Frequency Booster Pedal}

The schematic of an op-amp based "Frequency Booster" guitar pedal circuit is shown in Fig. 6a. It is based on a design by Anderton [52] (possibly via Hutchins [53], [54]) with additional biasing circuitry [54]. This circuit allows the user to add a parametric boost around one of five center frequencies. The circuit is essentially a type-16 Sallen-Key filter [55] with an extra dc coupling capacitor $\left(C_{\mathrm{z}}\right)$, extra biasing $\left(R_{0}, R_{\mathrm{a}}, R_{\mathrm{d}}, C_{\mathrm{a}}\right.$, and $\left.V_{\mathrm{b}}\right)$, and an extra high pass $\left(R_{\mathrm{g}}\right.$ and $C_{\mathrm{d}}$ ) on the output. The amplifier (op-amp, $R_{\mathrm{e}}, R_{\mathrm{f}}$, and $C_{\mathrm{c}}$ ) is not frequency-independent as in the traditional SallenKey design, but has a first-order high pass cutoff around $3 \mathrm{~Hz}$. The input is an ideal voltage source $v_{\text {in }}$ and the output is the voltage across $R_{\mathrm{g}}$. The circuit comprises 5 capacitors $\left(C_{\mathrm{z}}\right.$ and $\left.C_{\mathrm{a}} \cdots C_{\mathrm{e}}\right), 7$ resistors $\left(R_{0}, R_{\mathrm{a}}\right.$, and $\left.R_{\mathrm{c}} \cdots R_{\mathrm{h}}\right)$, a potentiometer $\left(R_{\mathrm{b}}\right)$ controlling the boost amount, an op-amp, and a battery (dc ideal voltage source $V_{\mathrm{b}}$ ). The op-amp is configured in negative feedback and modeled as ideal using a nullor [33].

1) WDF Model: A graph representing the circuit is shown in Fig. 6c. Circuit nodes correspond to graph nodes a $\cdots \mathrm{k}$ and electrical components correspond to graph edges $1 \cdots 16$; component values and mappings to graph edges are given in Fig. 6b. The unlabeled gray graph edges connected to nodes a, $\mathrm{f}, \mathrm{g}, \mathrm{j}$ are a replacement graph representing the op-amp (nullor) and keep this two-port contained inside a single junction (the same technique used for transformers in [26]). Using the graph separation algorithm of [26] yields a decomposition (Fig. 6d) into 5 series $\left(\mathcal{S}_{1} \cdots \mathcal{S}_{5}\right), 3$ parallel $\left(\mathcal{P}_{1} \cdots \mathcal{P}_{3}\right)$, and 1 rigid connection $\left(\mathcal{R}_{1}\right)$. Designating $v_{\text {in }}$ (edge 1 ) as the root, the graph decomposition yields an SPQR tree (Fig. 6e) isomorphic to a WDF adaptor structure (Fig. 6f) retaining the topology of the reference circuit (Fig. 6g). The series/parallel adaptors and one-ports of Fig. 6f are implemented using classical WDF techniques. The scattering behavior of $\mathcal{R}_{1}$ is derived using the proposed technique of this article and shown here in depth.

$\mathcal{R}_{1}$ involves an absorbed nullor and has six nodes (0)-(5) (Fig. 7a). Port voltages and currents for the 8 ports A $\cdots \mathrm{H}$ are labeled. Thévenin (Fig. 7b) or Norton (Fig. 7c) equivalents represent the incident waves $a_{\mathrm{A}} \cdots a_{\mathrm{H}}$. Using Thévenin equivalents, eight additional nodes (6)-(13) are added to the circuit. Using Norton Type 1 equivalents, the same is true but the extra nodes define shorts with currents $j_{\mathrm{A}} \cdots j_{\mathrm{H}}$. Using Norton Type 2 equivalents, these shorts are omitted.

Matrices $\mathbf{X}_{0}, \mathbf{X}_{1}$, and $\mathbf{A}_{\mathrm{p}}$ (Tab. VI) are formed by using 


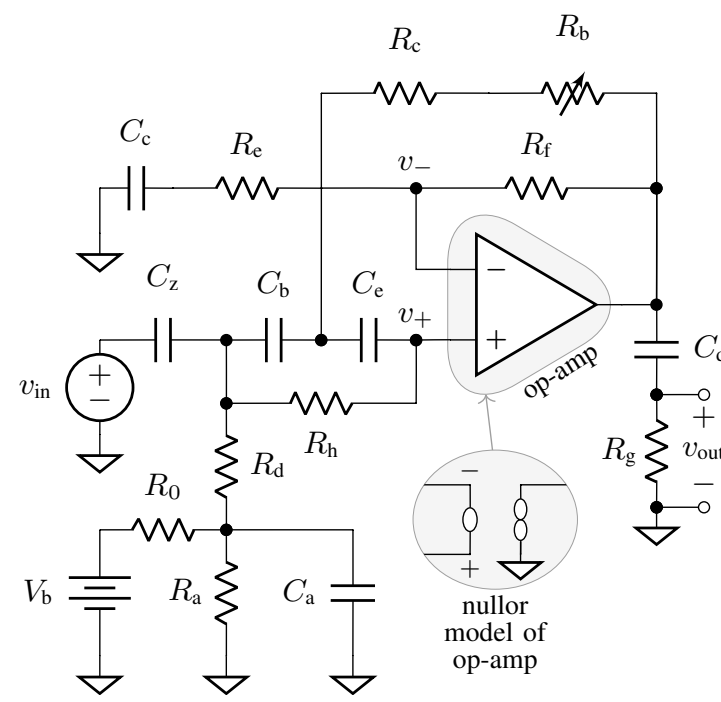

(a) Reference Circuit.

\begin{tabular}{lll}
\hline Value & Comp. & Edge \\
\hline input signal & $v_{\text {in }}$ & 1 \\
$100 \mathrm{k} \Omega$ & $R_{\mathrm{a}}$ & 4 \\
$10 \mathrm{k} \Omega$ & $R_{\mathrm{b}, \max }$ & -13 \\
$R_{\mathrm{b}, \max }(\mathrm{Q})$ & $R_{\mathrm{b}}$ & 14 \\
$5.6 \mathrm{k} \Omega$ & $R_{\mathrm{c}}$ & 6 \\
$1 \mathrm{M} \Omega$ & $R_{\mathrm{d}}$ & 11 \\
$10 \mathrm{k} \Omega$ & $R_{\mathrm{e}}$ & 12 \\
$10 \mathrm{k} \Omega$ & $R_{\mathrm{f}}$ & 16 \\
$100 \mathrm{k} \Omega$ & $R_{\mathrm{g}}$ & 7 \\
$10 \mathrm{k} \Omega$ & $R_{\mathrm{h}}$ & 3 \\
$9 \mathrm{~V}, 100 \mathrm{k} \Omega$ & $V_{\mathrm{b}}, R_{0}$ & 3 \\
$2.2 \mu \mathrm{F}$ & $C_{\mathrm{z}}$ & 2 \\
$10 \mu \mathrm{F}$ & $C_{\mathrm{a}}$ & 5 \\
$(\mathrm{C})$ & $C_{\mathrm{b}}$ & 8 \\
$10 \mu \mathrm{F}$ & $C_{\mathrm{c}}$ & 10 \\
$2.2 \mu \mathrm{F}$ & $C_{\mathrm{d}}$ & 15 \\
$(\mathrm{C})$ & $C_{\mathrm{e}}$ & 9 \\
\hline
\end{tabular}

(b) Graph Edges.

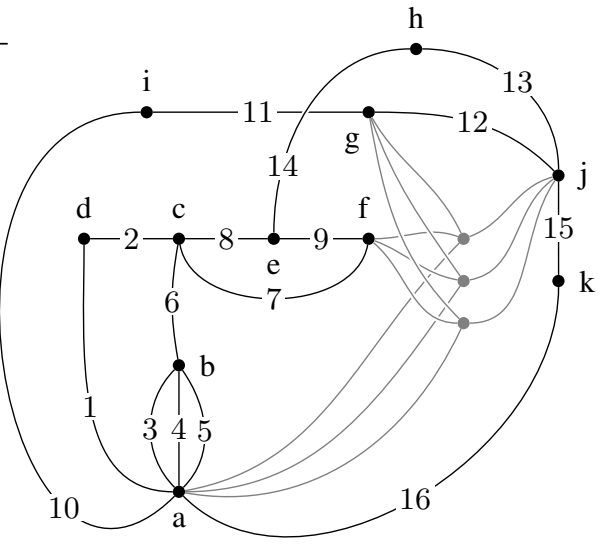

(c) Reference Circuit Graph.

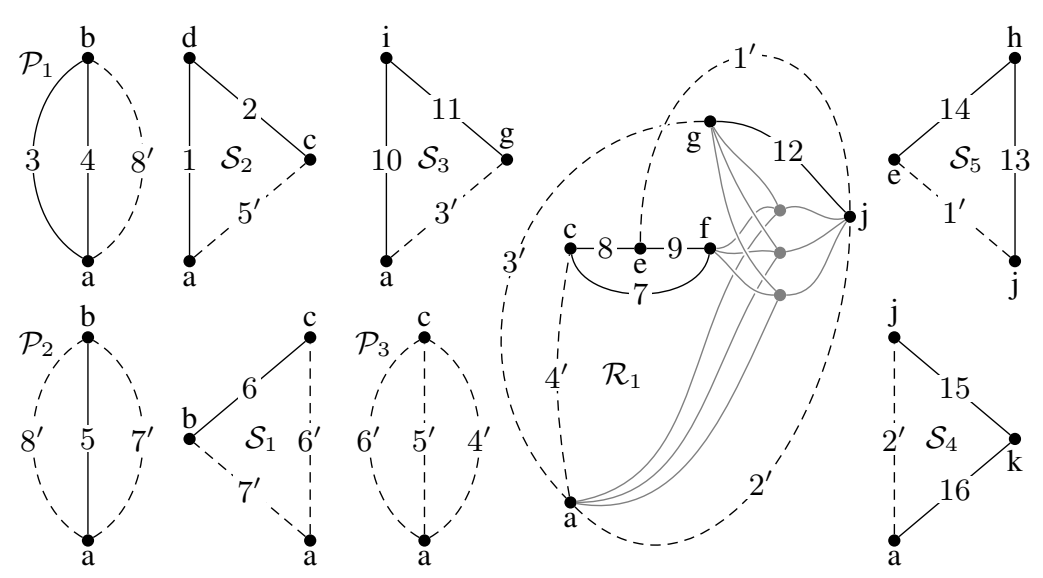

(d) Reference Circuit Graph Separated.

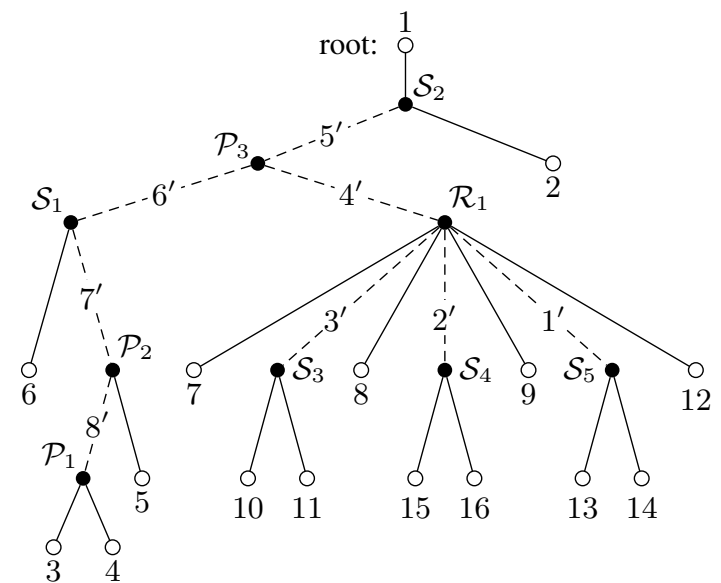

(e) SPQR Connection Tree (CT).

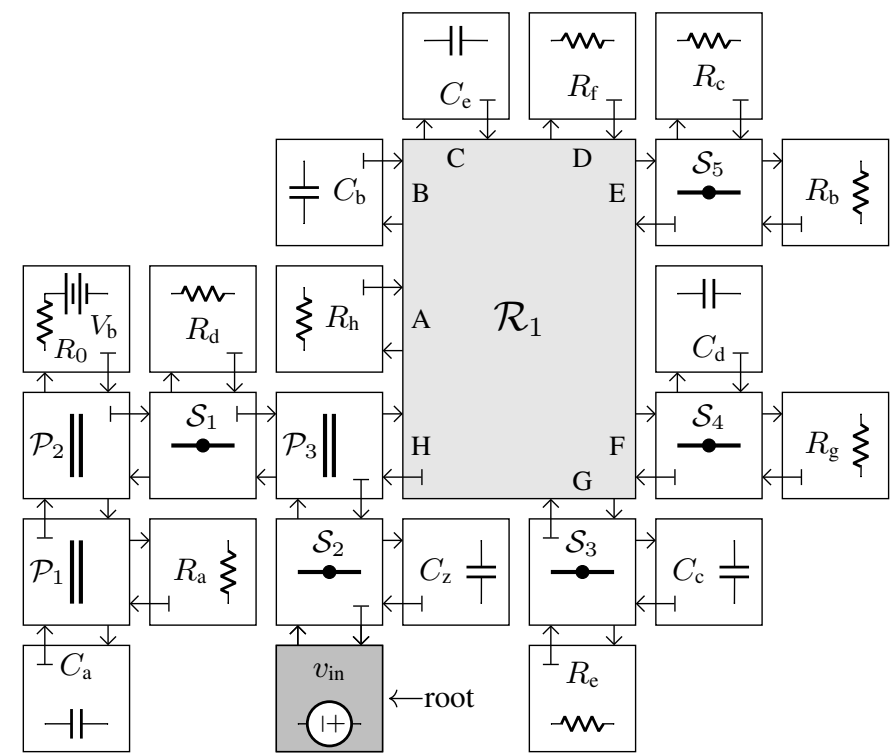

(f) Wave Digital Filter.

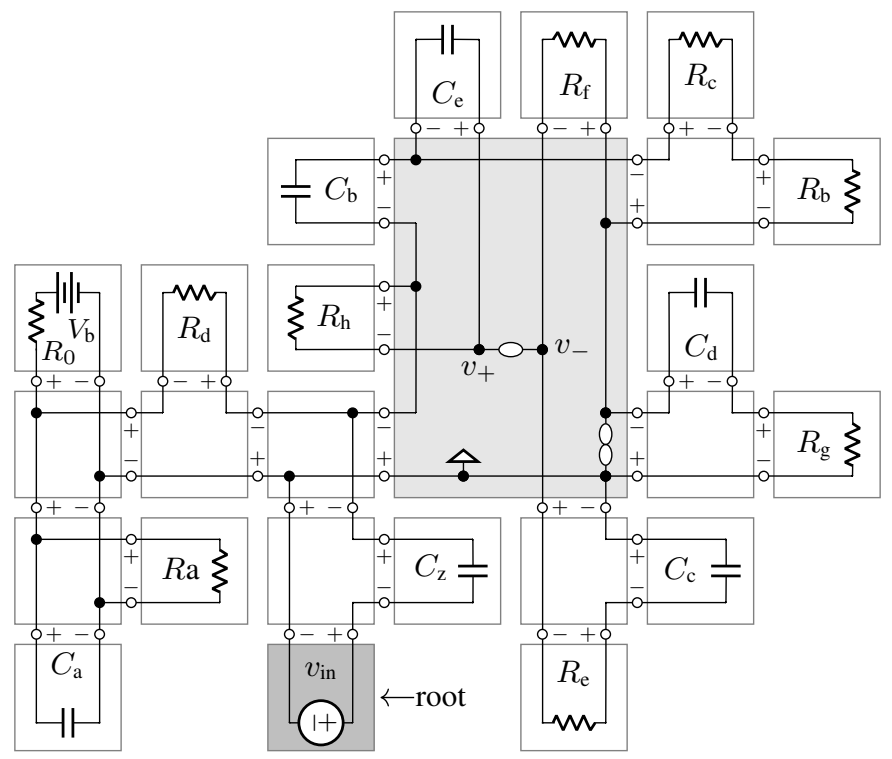

(g) Reference Circuit Rearranged.

Fig. 6. Steps for deriving Frequency Booster WDF simulation from schematic, including graph representation, graph separation, and SPQR steps. Quality factor "Q" control setting $\mathrm{Q} \in] 0,1]$. Capacitor pair value $\mathrm{C} \in\{0.22,0.1,0.02,0.01,0.005\} \mu \mathrm{F}$. 


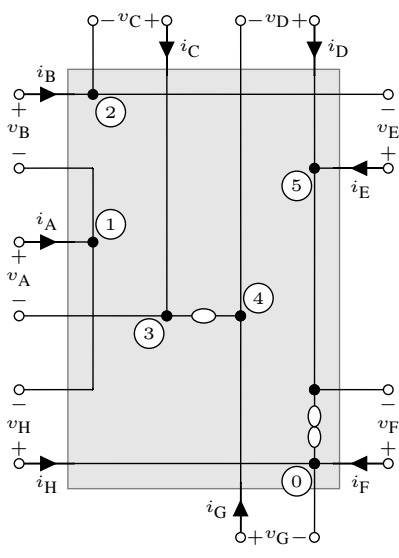

(a) $\mathcal{R}_{1}$ port definitions.

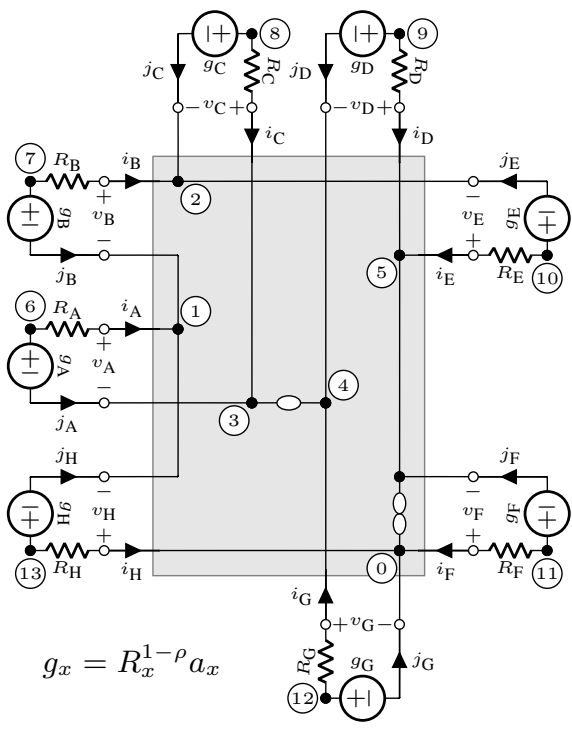

(b) $\mathcal{R}_{1}+$ Thévenin equivalents.

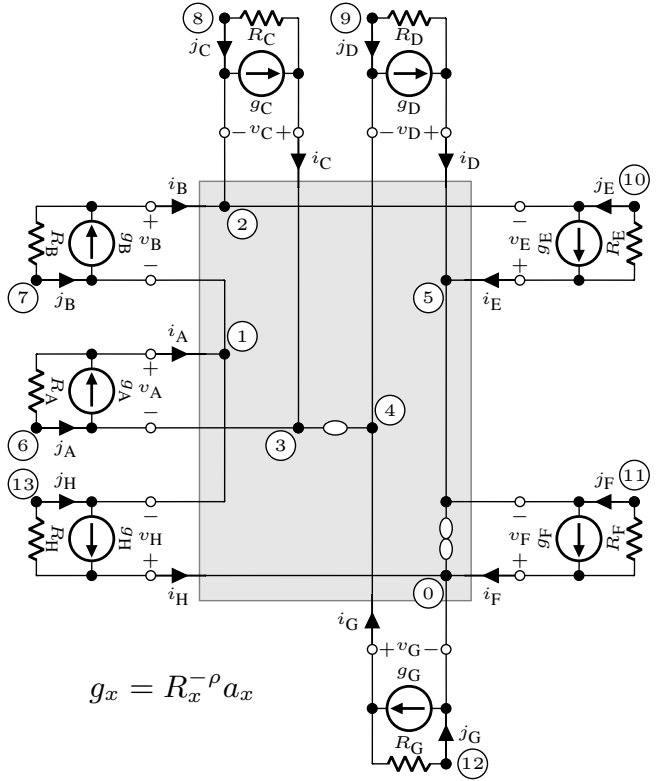

(c) $\mathcal{R}_{1}+$ Norton equivalents.

Fig. 7. $\mathcal{R}$-type junction definition for Frequency Booster and two versions of instantaneous port equivalents.

the Thévenin and Norton equivalent stamps from Tab. II and the nullor stamp from Tab. I. As an example, the stamps corresponding to port A are highlighted in light gray and the stamps corresponding to the nullor are highlighted in dark gray. Although we have chosen to model the op-amp with a nullor, consider modeling it with a VCVS as a thought experiment [33]. The procedure would be identical although the VCVS stamp from Tab. I would replace the nullor stamp.

In forming $\tilde{\mathbf{X}}_{0}$ or $\tilde{\mathbf{X}}_{1}$ and $\tilde{\mathbf{A}}_{\mathrm{p}}$ from $\mathbf{X}_{0}$ or $\mathbf{X}_{1}$ and $\mathbf{A}_{\mathrm{p}}$, any "internal" node (0)-(5) may be selected as the datum node as long as it is removed consistently from all matrices. As with other WDF adaptors, the upwards-facing port resistance $R_{\mathrm{H}}$ is chosen to adapt port $\mathrm{H}$ (i.e., to set $\mathbf{S}_{\mathrm{HH}}=0$ ). This is done by

$$
R_{\mathrm{H}}=\frac{R_{\mathrm{B}}\left(R_{\mathrm{A}} R_{\mathrm{D}}-R_{\mathrm{C}} R_{\mathrm{G}}\right)-\left(R_{\mathrm{A}}+R_{\mathrm{B}}+R_{\mathrm{C}}\right) R_{\mathrm{E}} R_{\mathrm{G}}}{\left(R_{\mathrm{A}}+R_{\mathrm{B}}+R_{\mathrm{C}}\right) R_{\mathrm{D}}} .
$$

2) Discussion: The model's frequency response $H=$ $V_{\text {out }} / V_{\text {in }}$ is characterized for a variety of boost and center frequency settings in Fig. 8, where nominal center frequencies $\left(C_{\mathrm{b}}\right.$ and $C_{\mathrm{e}}$ values) for each setting are shown with vertical lines. $R_{\mathrm{b}}$ cannot go to zero, i.e., $\left.\left.Q \in\right] 0,1\right]$. For the curves in Fig. 8, there is excellent agreement between the analog and WDF responses. The only visible error is high frequency bilinear transform warping. The boost control is weakly parametric; increasing the boost also alters the center frequency.

Since the Frequency Booster is an active circuit, it is possible that adaptation requires negative port resistances. Indeed, given the circuit values in Fig. 6b, that is the case for the upward-facing ports of $\mathcal{R}_{1}, \mathcal{P}_{3}$, and $\mathcal{S}_{2}$ : the three adaptors between the nullor and the root of the tree $\left(v_{\text {in }}\right)$. If the same wave definition is used in the entire WDF structure, the presence of negative port resistances would disallow the use of any wave type except voltage and current waves. An example of two strategies for localizing this restriction are shown in Fig. 9. On account of the negative port resistances, $\mathcal{P}_{3}, \mathcal{S}_{2}$,
$C_{\mathrm{z}}$, and $v_{\text {in }}$ all use voltage waves $(\rho=1)$. To interface this to a power waves $(\rho=1 / 2)$ part of the simulation, a wave converter is used between $\mathcal{S}_{1}$ and $\mathcal{P}_{3}$. $\mathcal{R}_{1}$ is treated using the "hybrid-wave junction" technique so that most of its ports can use power waves. Ports $A \cdots G$ use power waves and port $H$ uses voltage waves; $\mathcal{R}_{1}$ is parametrized by the vector

$$
\boldsymbol{\rho}=\left[\rho_{A}, \cdots, \rho_{G}, \rho_{H}\right]^{\top}=[1 / 2, \cdots, 1 / 2,1]^{\top} .
$$

In this way, the majority of the adaptors and one-ports in the WDF structure, including most of $\mathcal{R}_{1}$, can use power-waves despite the presence of negative port resistances.

Relevant quantities for analyzing $\mathcal{R}_{1}$ 's implementation cost in multiplies are shown in Tab. IV. The cost (from Tab. III) of (24)-(27) are shown for various wave types and compared against the nominal scattering matrix multiplication (20)(23) in Tab. V. The column " $\rho=$ else" is shown only to give a more complete sense of the computational impact of the wave parametrization; the negative port restrictions place some restrictions on wave definitions. The hybrid parametrization (32) can be used when power waves are preferred. The use of (27) (Nor.+Vol.) minimizes the number of multiplies needed, reducing the cost compared to the nominal scattering matrix multiplication by about $48.4 \%$ if voltage or current waves are used or $37.5 \%$ if (32) is used.

\section{CONClusion}

In this article, we expanded the class of circuits that can be modeled using the Wave Digital Filter approach to include active and non-reciprocal circuits. Beyond accomodating all of the topological ways that linear two-ports can be embedded in a circuit, this enables WDF modeling of circuits involving the important nullor element. Since the two-port scattering behavior of the nullor is undefined [22], they would previously have been intractable in a WDF simulation. 
TABLE VI

Frequency Booster $\mathcal{R}$-Type adaptor matrices $\mathbf{X}_{0}, \mathbf{X}_{1}$, ANd $\mathbf{A}_{\mathrm{P}}$ With Corrresponding Cases labelled.

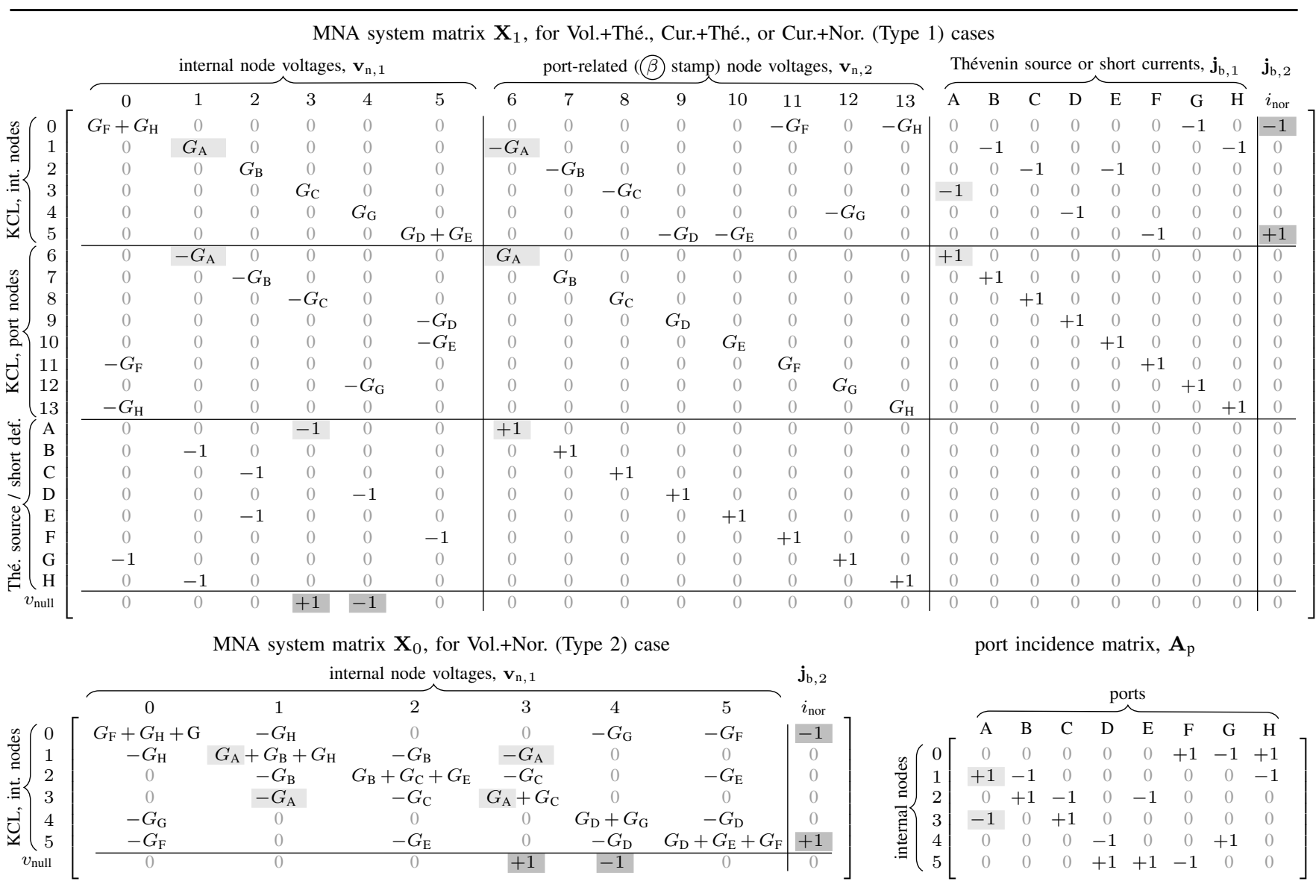

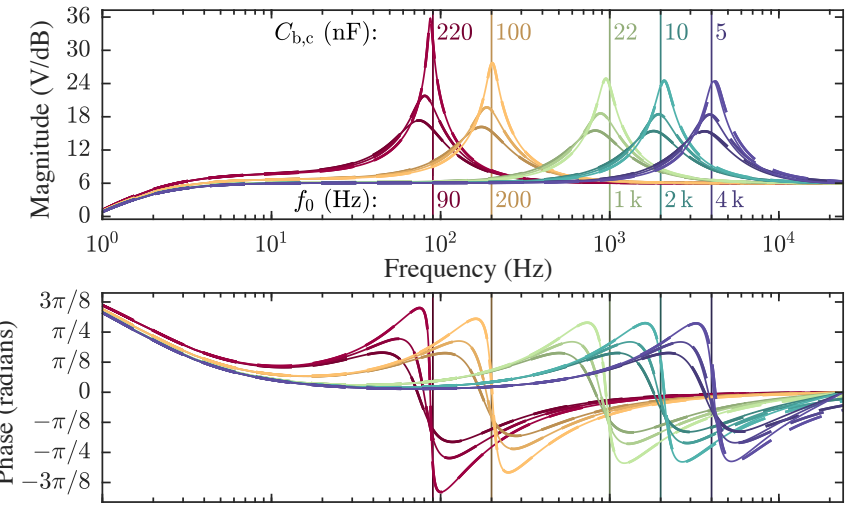

Fig. 8. Magnitude $|H|$ and phase $\angle H$ responses of Frequency Booster pedal.

First, a known technique [26] was shown to yield WDF structures involving $\mathcal{R}$-type adaptors for circuits with active linear elements. Second, four parametric-wave scattering equations were developed for the unbounded class of $\mathcal{R}$-type junctions. Third, three of these equations were shown to have implementation costs in multiplies that are usually cheaper than the cost of an $N \times N$ matrix multiply, with one (27) usually being the cheapest. Finally, wave converter two-ports and hybrid-wave junctions were introduced to enable multiple wave types to coexist, localizing restrictions on wave type.

The proposed methods do not introduce any loss of accuracy using infinite precision arithmetic. Although we have not experienced any numerical issues in practice, the proposed methods for $\mathcal{R}$-type adaptor scattering do not enjoy the same guarantee of pseudo-passivity as the classical basic WDF adaptors [1], [8]. Future work could investigate this detail.

The findings of this article have implications for WDF modeling of nonlinear circuits, which is a major topic [2], [12], [14], [17], [18], [43], [56]-[67]. In [68], Werner et al. showed one approach that groups all nonlinearities at the root of a tree and interfaces them to the rest of the circuit using an $\mathcal{R}$-type adaptor. The local implicit relationship that is created is resolved using, e.g., table lookup [68] or NewtonRaphson iteration [69]. This in theory enables systematic WDF simulation of circuits with any number of nonlinearities which were previously out of scope for WDF modeling, including the Electro-Harmonix Big Muff Pi [68], transistor [69] and triode [70] amplifiers, the Fender 5F6-A preamplifier [71], the Korg MS-50 VCF [72], relaxation oscillators [20], previously intractable types of diode clippers [73], and the Bass Drum circuit from the Roland TR-808 [39]. It also admits extensions for circuits with certain topological properties [73]. Future work should focus on incorporating the findings of this article into simulations of active, nonlinear circuits. 


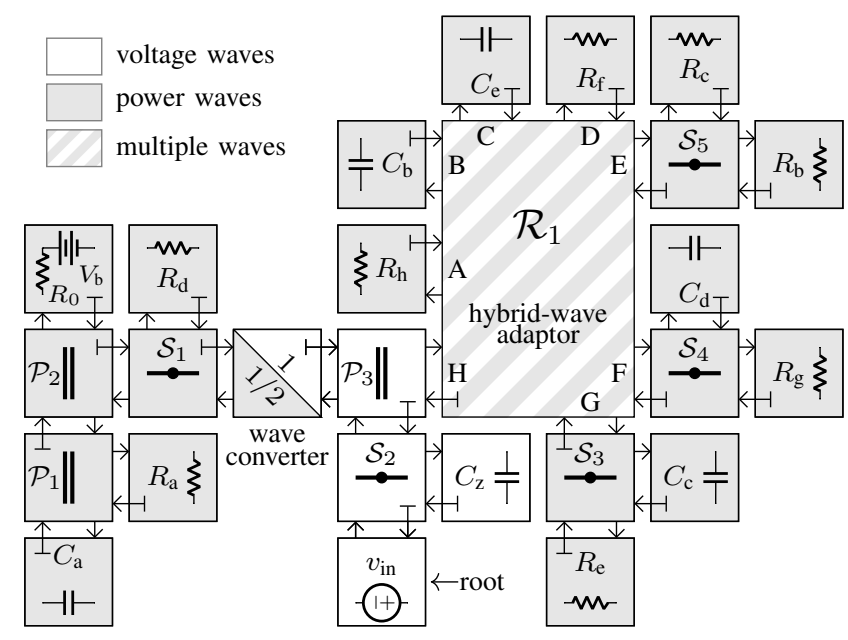

Fig. 9. Freq. Booster WDF with hybrid-wave adaptor and wave converter.

\section{REFERENCES}

[1] A. Fettweis, "Wave digital filters: Theory and practice," Proc. IEEE, vol. 74, no. 2, pp. 270-327, Feb. 1986.

[2] G. De Sanctis and A. Sarti, "Virtual analog modeling in the wave-digital domain," IEEE Trans. Audio, Speech, Language Process., vol. 18, no. 4, pp. 715-727, May 2010.

[3] J. O. Smith, Physical Audio Signal Processing. W3K Publishing, 2010.

[4] A. Fettweis, "Some principles of designing digital filters imitating classical filter structures," IEEE Trans. Circuit Theory, vol. 18, no. 2, pp. 314-316, Mar. 1971.

[5] - "Digital filters structures related to classical filter networks," Arch. Elek. Übertragung., vol. 25, pp. 79-89, Feb. 1971.

[6] V. Belevitch, Classical Network Theory. San Francisco, CA: HoldenDay, 1968.

[7] A. Fettweis and K. Meerkötter, "On adaptors for wave digital filters," IEEE Trans. Acoust., Speech, Signal Process., vol. 23, no. 6, pp. 516525, Dec. 1975.

[8] A. Fettweis, "Pseudo-passivity, sensitivity, and stability of wave digital filters," IEEE Trans. Circuit Theory, vol. 19, no. 6, pp. 668-673, Nov. 1972.

[9] A. Sedlmeyer and A. Fettweis, "Digital filter with true ladder configuration," Circuit Theory Appl., vol. 1, pp. 5-10, 1973.

[10] A. Fettweis, H. Levin, and A. Sedlmeyer, "Wave digital lattice filters," Circuit Theory Appl., vol. 2, pp. 203-211, 1974.

[11] J. O. Smith III, "Waveguide filter tutorial," in Proc. Int. Comput. Music Conf., Champaign-Urbana, IL, Aug. 1987, pp. 9-16.

[12] A. Sarti and G. De Poli, "Toward nonlinear wave digital filters," IEEE Trans. Signal Process., vol. 47, no. 6, pp. 1654-1668, June 1999.

[13] F. Pedersini, A. Sarti, and S. Tubaro, "Object-based sound synthesis for virtual environments using musical acoustics," IEEE Signal Process. Mag., vol. 17, no. 6, pp. 37-51, Nov. 2000.

[14] A. Sarti and G. De Sanctis, "Systematic methods for the implementation of nonlinear wave-digital structures," IEEE Trans. Circuits Syst. I, Reg. Papers, vol. 56, no. 2, pp. 460-472, Feb. 2009.

[15] A. Fettweis and G. Nitsche, "Numerical integration of partial differential equations by means of multidimensional wave digital filters," in Proc. IEEE Int. Symp. Circuits Syst., New Orleans, LA, May 1990, pp. 954 957.

[16] S. Bilbao, Wave and Scattering Methods for Numerical Simulation. New York, NY: Wiley, 2004.

[17] M. Karjalainen and J. Pakarinen, "Wave digital simulation of a vacuumtube amplifier," in IEEE Int. Conf. Acoust., Speech, Signal Process., Toulouse, France, May 2006, pp. 153-156.

[18] D. T.-M. Yeh, "Digital implementation of musical distortion circuits by analysis and simulation," Ph.D. diss., Stanford Univ., CA, June 2009.

[19] D. Fränken and K. Ochs, "Automatic step-size control in wave digital simulation using passive numerical integration methods," Int. J. Electron. Commun., vol. 58, pp. 391-401, 2004.

[20] M. J. Olsen, K. J. Werner, and F. G. Germain, "Network variable preserving step-size control in wave digital filters," in Proc. 20th Int. Conf. Digital Audio Effects, Edinburgh, UK, Sept. 2017, pp. 200-207.

[21] A. Vladimirescu, The SPICE Book. New York, NY: Wiley, 1994.
[22] H. J. Carlin, "Singular network elements," IEEE Trans. Circuit Theory, vol. 11, no. 1, pp. 67-72, Mar. 1964.

[23] L. T. Bruton, RC-Active Circuits: Theory and Design. Englewood Cliffs, NJ: Prentice-Hall, 1980.

[24] M. Karjalainen, "Efficient realization of wave digital components for physical modeling and sound synthesis," IEEE Trans. Audio, Speech, Language Process., vol. 16, no. 5, pp. 947-956, July 2008.

[25] K. J. Werner, J. O. Smith III, and J. S. Abel, "Wave digital filter adaptors for arbitrary topologies and multiport linear elements," in Proc. 18th Int. Conf. Digital Audio Effects, Trondheim, Norway, Nov. 2015, pp. 379386.

[26] D. Fränken, J. Ochs, and K. Ochs, "Generation of wave digital structures for networks containing multiport elements," IEEE Trans. Circuits Syst. I, Reg. Papers, vol. 52, no. 3, pp. 586-596, Mar. 2005.

[27] G. O. Martens and K. Meerkötter, "On N-port adaptors for wave digital filters with application to a bridged-tee filter," in Proc. IEEE Int. Symp. Circuits Syst., Munich, Germany, Apr. 1976, pp. 514-517.

[28] H. H. Lê, "Wave digital adaptors for Brune, Darlington C and D, and twin-T sections," Ph.D. diss., Univ. Manitoba, Winnipeg, Canada, 1977.

[29] G. O. Martens and H. H. Lê, "Wave digital adaptors for reciprocal second-order sections," IEEE Trans. Circuits Syst., vol. 25, no. 12, pp. 1077-1083, Dec. 1978.

[30] K. Meerkötter and D. Fränken, "Digital realization of connection networks by voltage-wave two-port adaptors," Arch. Elek. Übertragung., vol. 50, no. 6, pp. 362-367, 1996.

[31] K. J. Werner, V. Nangia, J. O. Smith III, and J. S. Abel, "A general and explicit formulation for wave digital filters with multiple/multiport nonlinearities and complicated topologies," in Proc. IEEE Work. Appl. Signal Process. Audio Acoust., New Paltz, NY, Oct. 2015.

[32] C.-W. Ho, A. E. Ruehli, and P. A. Brennan, "The modified nodal approach to network analysis," IEEE Trans. Circuits Syst., vol. 22, no. 6, pp. 504-509, June 1975.

[33] K. J. Werner, W. R. Dunkel, M. Rest, M. J. Olsen, and J. O. Smith III, "Wave digital filter modeling of circuits with operational amplifiers," in Proc. Eur. Signal Process. Conf., Budapest, Hungary, Aug. 2016, pp. 1033-1037.

[34] K. J. Werner, W. R. Dunkel, and F. G. Germain, "A computational model of the Hammond organ vibrato/chorus using wave digital filters," in Proc. 19th Int. Conf. Digital Audio Effects, Brno, Czech Republic, Sept. 2016, pp. 271-278.

[35] F. G. Germain and K. J. Werner, "Optimizing differentiated discretization for audio circuits beyond driving point transfer functions," in Proc. IEEE Work. Appl. Signal Process. Audio Acoust., New Paltz, NY, Oct. 2017.

[36] Ó. Bogason, "Digitizing analog circuits containing op amps using wave digital filters," Online Blog, URL: http://obogason.com/emulating-opamp-circuits-using-wdf-theory/, Mar. 202016.

[37] M. Verasani, A. Bernardini, and A. Sarti, "Modeling Sallen-Key audio filters in the wave digital domain," in Proc. IEEE Int. Conf. Acoust., Speech Signal Process., New Orleans, LA, Mar. 2017, pp. 431-435.

[38] Ó. Bogason and K. J. Werner, "Modeling circuits with operational transconductance amplifiers using wave digital filters," in Proc. 20th Int. Conf. Digital Audio Effects, Edinburgh, UK, Sept. 2017, pp. 130-137.

[39] K. J. Werner, "Virtual analog modeling of audio circuitry using wave digital filters," Ph.D. diss., Stanford Univ., CA, Dec. 2016.

[40] G. Kubin, "Wave digital filters: Voltage, current, or power waves?" in IEEE Int. Conf. Acoust., Speech, Signal Process., Tampa, FL, Apr. 1985, pp. 69-72.

[41] S. D'Angelo and V. Välimäki, "Wave-digital polarity and current inverters and their application to virtual analog audio processing," in IEEE Int. Conf. Acoust., Speech Signal Process., Kyoto, Japan, Mar. 2012.

[42] J. Vlach and K. Singhal, Computer Methods for Circuit Analysis and Design. New York, NY: Van Nostrand Reinhold Company, 1983.

[43] A. Bernardini, P. Maffezzoni, L. Daniel, and A. Sarti, "Wave-based analysis of large nonlinear photovoltaic arrays," IEEE Trans. Circuits Syst. I, Reg. Papers, vol. 65, no. 4, pp. 1363-1376, Apr. 2018.

[44] I. M. Gottlieb, Practical Oscillator Handbook. Oxford, UK: Newnes, 1997.

[45] P. J. Baxandall, "Negative-feedback tone control," Wireless World, pp. 402-405, Oct. 1952.

[46] V. Välimäki and J. D. Reiss, "All about audio equalization: Solutions and frontiers," Appl. Sci., vol. 6, no. 5, 2016, article \#129.

[47] D. Self, Small Signal Audio Design. Oxford, UK: Focal Press, 2010.

[48] V. Välimäki, S. Bilbao, J. O. Smith III, J. S. Abel, J. Pakarinen, and D. Berners, "Virtual analog effects," in DAFx: Digital Audio Effects, 2nd ed., U. Zölzer, Ed. West Sussex, UK: Wiley, 2011, ch. 12, pp. 473-522. 
[49] M. Giles, Ed., Audio/Radio Handbook. Santa Clara, CA: National Semiconductor Corp., 1980.

[50] F. G. Germain and K. J. Werner, "Design principles for lumped model discretisation using Möbius transforms," in Proc. 18th Int. Conf. Digital Audio Effects, Trondheim, Norway, Nov. 2015, pp. 371-378.

[51] D. T.-M. Yeh and J. O. Smith III, "Discretization of the '59 Fender Bassman tone stack," in Proc. 9th Int. Conf. Digital Audio Effects, Montréal, Canada, Sept. 2006, pp. 1-5.

[52] C. Anderton, Projects for Guitarists. San Francisco, CA: Miller Freeman Books, 1995, Project 10: The Frequency Booster.

[53] B. Hutchins, "A simple mid-range booster," Electronotes Application Notes, vol. AN-215, May 1981.

[54] J. Orman, "Anderton frequency booster," Online Blog, URL: http://www.muzique.com/news/frequency-booster/, Nov. 2010.

[55] R. P. Sallen and E. L. Key, "A practical method of designing RC active filters," IRE Trans. Circuit Theory, vol. 2, no. 1, pp. 74-85, Mar. 1955.

[56] K. Meerkötter and R. Scholz, "Digital simulation of nonlinear circuits by wave digital filter principles," in IEEE Int. Symp. Circuits Syst., Portland, OR, May 1989, pp. 720-723.

[57] S. Petrausch and R. Rabenstein, "Wave digital filters with multiple nonlinearities," in Proc. Eur. Signal Process. Conf., Vienna, Austria, Sept. 2004, pp. 77-80.

[58] R. C. D. Paiva, S. D'Angelo, J. Pakarinen, and V. Välimäki, "Emulation of operational amplifiers and diodes in audio distortion circuits," IEEE Trans. Circuits Syst. II, Exp. Briefs, vol. 59, no. 10, pp. 688-692, Oct. 2012.

[59] K. J. Werner, V. Nangia, A. Bernardini, J. O. Smith III, and A. Sarti, "An improved and generalized diode clipper model for wave digital filters," in Proc. 139th Conv. Audio Eng. Soc., New York, NY, Oct. 2015, conv. paper. \#9360.

[60] T. Schwerdtfeger and A. Kummert, "A multidimensional approach to wave digital filters with multiple nonlinearities," in Proc. Eur. Signal Process. Conf., Lisbon, Portugal, Sept. 2014, pp. 2405-2409.

[61] — - "Newton's method for modularity-preserving multidimensional wave digital filters," in Proc. IEEE Int. Work. Multidimensional Syst., Vila Real, Portugal, Sept. 2015

[62] A. Bernardini, K. J. Werner, A. Sarti, and J. O. Smith III, "Modeling nonlinear wave digital elements using the Lambert function," IEEE Trans. Circuits Syst. I, Reg. Papers, vol. 63, no. 8, pp. 1231-1242, Aug. 2016.

[63] Ó. B. Bogason, "Modeling audio circuits containing typical nonlinear components with wave digital filters," Master's thesis, McGill Univ., Montreal, Canada, Apr. 2018.

[64] A. Bernardini and A. Sarti, "Biparametric wave digital filters," IEEE Trans. Circuits Syst. I, Reg. Papers, vol. 64, no. 7, pp. 1826-1838, July 2017.

[65] _ - "Dynamic adaptation of instantaneous nonlinear bipoles in wave digital networks," in Proc. Eur. Signal Process. Conf., Budapest, Hungary, Aug. 2016, pp. 1038-1042.

[66] - "Canonical piecewise-linear representation of curves in the wave digital domain," in Proc. Eur. Signal Process. Conf., Kos, Greece, Aug. 2017, pp. 1125-1129.

[67] A. Bernardini, K. J. Werner, P. Maffezzoni, , and A. Sarti, "Wave digital modeling of the diode-based ring modulator," in Proc. 144nd Conv. Audio Eng. Soc., Milan, Italy, May 2018, conv. paper \#10015.

[68] K. J. Werner, V. Nangia, J. O. Smith III, and J. S. Abel, "Resolving wave digital filters with multiple/multiport nonlinearities," in Proc. 18th Int. Conf. Digital Audio Effects, Trondheim, Norway, Nov. 2015, pp. 387-394.

[69] M. J. Olsen, K. J. Werner, and J. O. Smith III, "Resolving grouped nonlinearities in wave digital filters using iterative techniques," in Proc. 19th Int. Conf. Digital Audio Effects, Brno, Czech Republic, Sept. 2016, pp. 279-286.

[70] M. Rest, W. R. Dunkel, K. J. Werner, and J. O. Smith III, "RT-WDF-a modular wave digital filter library with support for arbitrary topologies and multiple nonlinearities," in Proc. 19th Int. Conf. Digital Audio Effects, Brno, Czech Republic, Sept. 2016, pp. 287-294.

[71] W. R. Dunkel, M. Rest, K. J. Werner, M. J. Olsen, and J. O. Smith III, "The Fender Bassman 5F6-A family of preamplifier circuits-a wave digital filter case study," in Proc. 19th Int. Conf. Digital Audio Effects, Brno, Czech Republic, Sept. 2016, pp. 263-270.

[72] M. Rest, J. D. Parker, and K. J. Werner, "WDF modeling of a Korg MS-50 based non-linear diode bridge VCF," in Proc. 20th Int. Conf. Digital Audio Effects, Edinburgh, UK, Sept. 2017, pp. 145-151.

[73] K. J. Werner, M. J. Olsen, M. Rest, and J. D. Parker, "Root variable choice in grouped wave digital filters," in Proc. 20th Int. Conf. Digital Audio Effects, Edinburgh, UK, Sept. 2017, pp. 176-183.

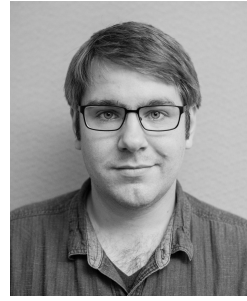

Kurt James Werner (S'14-M'17) received his B.S. (2011) in General Engineering (with a secondary field in Acoustics) and B.Music in Theory and Composition, both from the University of Illinois at Urbana-Champaign (UIUC). He earned a Ph.D. (2017) in Computer-Based Music Theory and Acoustics from Stanford University's Center for Computer Research in Music and Acoustics. He is a Lecturer in Audio at the Sonic Arts Research Centre (SARC) of Queen's University Belfast, where he joined the faculty of Arts, Humanities and Social Sciences and the School of Arts, English and Languages in 2017. His research focuses on theoretical aspects of wave digital filters, virtual analog modeling, computer modeling of circuit-bent instruments, sound synthesis, and the history of music technology. Dr. Werner is a member of the Audio Engineering Society (AES) and the International Computer Music Association (ICMA). He has been awarded best student paper awards from the IEEE WASPAA (2015) and (as second author) AES (2017). As co-author he was also awarded best paper no. 3 (DAFx, 2016) and a best paper honorable mention (DAFx, 2015).

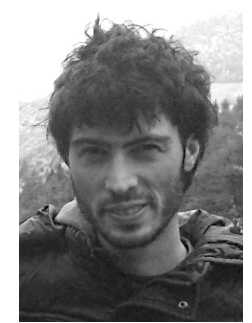

Alberto Bernardini (S'16) received his B.S. from the University of Bologna, Italy, in 2012; and his M.S. degree (cum laude) from the Politecnico di Milano, Italy, in 2015, both in Computer Engineering. He is currently a Ph.D. candidate in Information Engineering at the Dipartimento di Elettronica, Informazione and Bioingegneria of the Politecnico di Milano, Italy. His main research interests are audio signal processing and modeling of nonlinear systems.

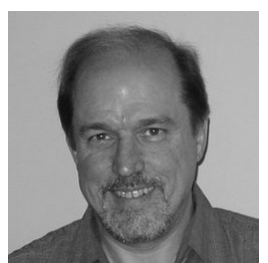

Julius Orion Smith III (M'76) received the B.S.E.E. degree from Rice University, Houston, TX, in 1975 (control, circuits, and communication). $\mathrm{He}$ received the M.S. and Ph.D. degrees in E.E. from Stanford University, Stanford, CA, in 1978 and 1983, respectively. His Ph.D. research was devoted to improved methods for digital filter design and system identification applied to music and audio systems. From 1975 to 1977 , he worked in the Signal Processing Department at ESL, Sunnyvale, $\mathrm{CA}$, on systems for digital communications. From 1982 to 1986, he was with the Adaptive Systems Department at Systems Control Technology, Palo Alto, CA, where he worked in the areas of adaptive filtering and spectral estimation. From 1986 to 1991, he was employed at NeXT Computer, Inc., responsible for sound, music, and signal processing software for the NeXT computer workstation. After NeXT, he became an Associate Professor at the Center for Computer Research in Music and Acoustics (CCRMA) at Stanford, teaching courses and pursuing research related to signal processing techniques applied to music and audio systems. Continuing this work, he is presently Professor of Music and (by courtesy) Electrical Engineering at Stanford University. For more information, see http://ccrma.stanford.edu/ jos/.

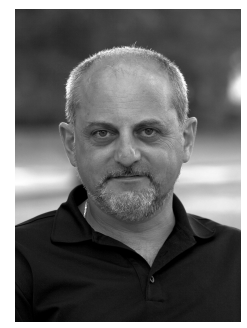

Augusto Sarti (M'04-SM'13) received his M.S. and his $\mathrm{Ph} . \mathrm{D}$. in Electronics and Information Engineering from the University of Padua, Italy, in 1988 and 1993, respectively. In 1993, he joined the faculty of the Politecnico di Milano, Italy, where he is currently a full professor. In 2013, he also joined the University of California, Davis. His research interests are in the area of multimedia signal processing, with particular focus on sound analysis, synthesis and processing; computational acoustics; and music processing. He coauthored over 250 scientific publications on international journals and congresses as well as numerous patents in the multimedia signal processing area. He coordinates the activities of the Musical Acoustics Lab and of the Sound and Music Computing Lab of the Politecnico di Milano. He has been the promoter/coordinator and/or contributor to numerous (20+) European projects. He is an active member of the IEEE TC on Audio and Acoustics Signal Processing, and is in the Editorial Board of the IEEE. 